\title{
REFLEXIONES SOBRE LA IMPLEMENTACIÓN DE LA POLÍTICA PÚBLICA DE PREVENCIÓN DE VIOLACIONES DE DERECHOS HUMANOS Y DIH EN COLOMBIA: UN BREVE RECUENTO HISTÓRICO DE SU IMPLEMENTACIÓN DESDE 2001 $-2010$
}

Luis Milciades Pérez González ${ }^{1}$

\section{Resumen}

Este artículo tiene como objetivo ilustrar cómo ha sido el proceso de implementación de la política pública de prevención de violaciones de derechos humanos durante el periodo 2001 al 2010. Para llevar a cabo el análisis de la implementación de ésta política, se utilizó un enfoque integrado propuesto por Soren Winter (1990) a partir del estudio de cuatro elementos: el proceso de formulación de la política, el comportamiento organizacional e interorganizacional, el papel de los burócratas "de primer nivel" y la reacción de los grupos destinatarios y los cambios en el contexto social. Se deduce como resultado del análisis, que en Colombia aún existen fuertes barreras para una eficaz implementación, donde los comportamientos organizacionales y de los funcionarios públicos (burócratas de primer nivel), determinan en gran manera el tipo de resultado de la política, y que por tanto explican el actual estado del riesgo de violaciones de derechos humanos e infracciones al Derecho Internacional Humanitario -DIH-.

1 Economista, Magister en Gobierno. Servidor Público, Delegado para la prevención de riesgos y Sistemas de Alertas Tempranas de la Defensoría del Pueblo. Email: luperez@defensoria.gov.co 

de derechos humanos y DIH en Colombia: un breve recuento...

Palabras clave: Políticas públicas, implementación de políticas públicas, derechos humanos, prevención, violaciones de derechos humanos.

\section{Abstract}

This article aims to illustrate the process of implementation of the public policy of prevention of human rights violations during the period 2001 to 2010. To carry out the analysis of the implementation of this policy, an integrated approach proposed by Soren Winter (1990) was used, based on the study of four elements: the process of policy formulation, organizational and interorganizational behavior, the role of "first level" bureaucrats, and the reaction of target groups and changes in the social context. As a result of the analysis, it can be deduced that in Colombia there are still strong barriers to an effective implementation, where the organizational behaviors and those of public officials (first level bureaucrats), determine to a great extent the type of result of the policy, and therefore explain the current state of risk of human rights violations and breaches of International Humanitarian Law (IHL).

Key words: Public policies, implementation of public policies, human rights, prevention, human rights violations. 


\section{Introducción}

¿Cómo implementar una política pública de prevención en un país que se encuentra inmerso en un conflicto armado, donde la población civil es la más victimizada? Esta es una de las principales preguntas que surgió desde mediados de los años noventa en las autoridades colombianas que observaban con estupor y resignación el incremento de masacres, desplazamientos masivos, enfrentamientos con interposición civil y por tanto homicidios, desapariciones forzadas y accidentes.

Adicionalmente, ¿Cómo el diseño institucional, la formulación de la política, el rol de los actores responsables de la implementación, los grupos objetivos y el contexto territorial, afectan el cumplimiento de los objetivos de un programa de prevención y protección de derechos humanos e infracciones al Derecho Internacional Humanitario en el marco del conflicto armado interno en Colombia?

Este artículo centra su atención en el proceso de configuración de lo que puede denominarse como política pública de prevención de violaciones masivas de derechos humanos a comienzos del año 2001, cuyo objetivo principal buscaba prevenir las reiteradas masacres y desplazamientos masivos de población y demás infracciones al Derecho Internacional Humanitario -DIH- cometidos por los actores armados ilegales en el marco del conflicto armado interno, a través de la implementación del Sistema General de Alertas Tempranas (SGAT).

El SGAT para la prevención de violaciones masivas de derechos humanos, lo conforman el Sistema de Alertas Tempranas (SAT) de la Defensoría del Pueblo y la Comisión Intersectorial de Alertas Tempranas (CIAT) del Ministerio del Interior y de Justicia. Ambas instancias configuran el eje de la prevención en el Estado colombiano; sin embargo, desde su creación, es muy poco lo que se sabe sobre el proceso de implementación de dicho Programa.

Para aproximarse a la comprensión de éste proceso, debemos preguntarnos ¿Cómo ha sido el proceso de implementación de la política pública de prevención de violaciones de derechos humanos durante el periodo 2001 - 2010? A partir de la respuesta de este interrogante se espera comprender. ¿Qué factores determinan la incidencia y eficacia de las acciones 

de derechos humanos y DIH en Colombia: un breve recuento...

en materia de prevención de violaciones de DDHH e infracciones al DIH? Metodológicamente el estudio se orienta a partir del enfoque integrado de Soren Winter (1990).

El artículo se divide en tres secciones. La primera sección explica el marco teórico del análisis del proceso de implementación de las políticas públicas; se utiliza como metodología de análisis el modelo de Soren Winter; para ello describen los elementos del modelo y su respectiva definición en cada una de las fases del proceso de implementación de una política pública.

La segunda sección describe el proceso histórico de la implementación del SAT/CIAT, abordando sus vicisitudes políticas durante su surgimiento y creación. Se incluyen algunos resultados de dos evaluaciones hechas por MSI (2004) y Democracia Internacional (2009), ambas solicitadas por la USAID a su programa de Derechos Humanos en Colombia.

En la tercera sección se abordan algunos resultados del análisis, descritos según los cuatro elementos del enfoque integrado de Winter. Cada elemento de este modelo se desarrolla considerando la eficacia de la implementación y de los objetivos de la política de prevención de violaciones de derechos humanos.

Se espera que los resultados de esta investigación contribuyan a la reflexión sobre la política de derechos humanosy de prevención de violaciones de DDHH existente en el País y la manera como se diseñan e implementan. Para ello se ha considerado que un buen ejemplo para su análisis es el sistema SAT/CIAT que ha venido tomando resonancia política en Colombia.

\section{Sección primera. El ciclo de las políticas públicas: la implementación}

La implementación designa la fase de una política durante la cual se generan actos y efectos a partir de un marco normativo de intenciones, de textos o de discursos (Meny y Thoenig, 1992; pág. 158). "Todas las instituciones involucradas en un proceso de política pública -o administración, parlamento, ejecutivos, gremios, etc. - tienen características sociales y políticas e intereses que hacen de cada una de ellas un actor más del juego político administrativo". Así mismo, "La implementación se 
revela como un escenario en que intervienen actores que no permanecen neutros ni pasivos" (Meny y Thoenig, 1992; pág. 164). En síntesis, "los ejecutores adoptan posiciones frente al tema: distracción de los recursos, deformación de los objetivos, elusión de los controles exteriores, uso de la ley para aplicarla a otras políticas públicas". (...) "La ambigüedad de los textos y la flexibilidad de las disposiciones extienden el juego político hasta el ejecutor" (Meny y Thoenig, 1992; pág. 166).

Al considerar estos criterios en el análisis dentro del proceso de construcción de la política pública de derechos humanos, se puede observar que son muchos los obstáculos e intereses que subyacen a la concreción de la misma, y que cobran mayor interés cuando la teoría causal está relacionada con la aplicación del enfoque de los DDHH en las políticas públicas de seguridad y prevención.

Lo anterior conlleva revisar el abordaje de los enfoques para el estudio de la implementación de políticas y programas públicos. De acuerdo con Ryan (1996), "La literatura sobre la gestión del sector público ha tendido a basarse en los modelos racionales de conjunto para interpretar los resultados del desarrollo en los procesos. Estos modelos de análisis de implementación de políticas y programas públicos, se han caracterizado por una tensión entre los enfoques de gestión pública del "top-down” Esta perspectiva, en la literatura anglosajona, "describe la puesta en práctica como una secuencia lineal que desciende del centro hacia la periferia. Lo alto gobierna por la definición del sentido y de los fines, y por el mantenimiento de la autoridad. La base aplica, por conformidad a la jerarquía y por apropiación instrumental. (...) El paso del centro a la periferia se traduce por la transformación de los objetivos en medios, por la sustitución de la política por la técnica, por la desaparición de los desafíos conflictivos en provecho de las racionalidades gestionarías." Meny y Thoenig, 1992; pág. 159-160. y del "bottom-up" Según este enfoque:

Se trata de partir de los comportamientos concretos en el nivel donde existe el problema para construir la política pública poco a poco, con reglas, procedimientos y estructuras organizativas por medio de un proceso ascendente, o por retroceso, en vez de descendente.

El investigador Hjern, es el primero que basado en la teoría del public choice, propone un enfoque bottom-up, que vaya de abajo a arriba 

de derechos humanos y DIH en Colombia: un breve recuento...

(Hjern, Porter, 1980). Este modelo se ve enriquecido por los aportes de El more (1987) quien propone por su parte "un razonamiento de retroceso o backward mapping, que aplica a las políticas de empleo para la juventud. (Roth, 2007)... El primero de ellos corresponde a la concepción tradicional del trabajo administrativo que se desarrolla de arriba (top) hacia abajo (Down); tiene como postulados principales la primacía jerárquica de la autoridad, la distinción entre el universo político y el mundo administrativo y, por último, la búsqueda del principio de eficiencia (Meny, Thoenig; 1992, 159). El marco de análisis más utilizado ha sido el modelo de aplicación de Mazmanian \& Sabatier (1989). Sin embargo, el enfoque top-Down aplicado al análisis de la implementación de la gestión pública, tiene una capacidad limitada para considerar problemas de bottom-up como la participación de los burócratas de "primer nivel o de calle" y los grupos del programa/ objetivo (véase Sabatier 1986, citado por Ryan).

El modelo cíclico de la política pública tiene como marco de interpretación legalista de la acción pública (perspectiva top-down) centrarse en la acción del Estado, y no toma en cuenta la perspectiva según la cual el punto de partida son los actores sociales y su contexto (perspectiva bottom-up). En el siguiente Tabla 1 se sintetizan las diferencias entre los dos enfoques en la etapa de implementación de políticas públicas.

Gogginetal.(1990)muestracomodesdehacemásveinteañosseprodujo una "tercera generación" de teorías que tratan de unificar el pluralismo teórico que se desarrolla desde principios de top-down y del bottom-up, en el análisis de la implementación. Un enfoque reciente en la consolidación de los elementos clave del "top-down" y la literatura del "bottom-up", es el proporcionado por Soren C. Winter (1990), conocido como Enfoque Integrado, el cual muestra que en los modelos de uso racional aplicados al análisis de la implementación, como el marco de Mazmanian y Sabatier, se presentan algunas debilidades. Sin embargo, estas conceptualizaciones, top-down y bottom-up, proporcionan un medio conveniente para incorporar enfoques integrados dentro de la gama de teorías de la implementación de políticas públicas. 
Tabla 1

Las visiones top-down y bottom-up en la implementación de una política pública

\begin{tabular}{|c|c|c|}
\hline $\begin{array}{c}\text { Enfoque de } \\
\text { implementación }\end{array}$ & $\begin{array}{c}\text { Visión Top-down } \\
\text { (Sabatier \& Mazmanian, } \\
\text { 1979) }\end{array}$ & $\begin{array}{c}\text { Visión Bottom-up } \\
\text { (Hjern \& Hull, 1982) }\end{array}$ \\
\hline $\begin{array}{l}\text { Punto de partida } \\
\text { del análisis de la } \\
\text { implementación de la } \\
\text { política pública }\end{array}$ & $\begin{array}{l}\text { Decisiones de las } \\
\text { autoridades político- } \\
\text { administrativas (PPA, } \\
\text { Planes de Acción) }\end{array}$ & $\begin{array}{l}\text { Actividades de la red de } \\
\text { implementación a nivel } \\
\text { local (APA, red de acción } \\
\text { pública) }\end{array}$ \\
\hline $\begin{array}{l}\text { Proceso para la } \\
\text { identificación de los } \\
\text { principales actores de } \\
\text { la política pública }\end{array}$ & $\begin{array}{l}\text { Desde arriba, y a partir } \\
\text { del sector público, hacia } \\
\text { abajo, y hacia el sector } \\
\text { privado }\end{array}$ & $\begin{array}{l}\text { Desde abajo (street-level } \\
\text { actors), hacia arriba, } \\
\text { considerando de manera } \\
\text { simultánea los actores } \\
\text { públicos y privados }\end{array}$ \\
\hline \multirow{3}{*}{$\begin{array}{l}\text { Criterios de } \\
\text { evaluación de } \\
\text { la calidad de la } \\
\text { implementación de la } \\
\text { política pública }\end{array}$} & $\begin{array}{l}\text { Regularidad (conformidad } \\
\text { legal) del proceso de } \\
\text { implementación }\end{array}$ & $\begin{array}{l}\text { No tiene criterios } \\
\text { claramente definidos a } \\
\text { priori }\end{array}$ \\
\hline & \multirow[t]{2}{*}{$\begin{array}{l}\text { Eficacia: grado de } \\
\text { realización de los objetivos } \\
\text { formales del PPA }\end{array}$} & $\begin{array}{l}\text { Evaluación del grado de } \\
\text { participación de los actores } \\
\text { involucrados }\end{array}$ \\
\hline & & $\begin{array}{l}\text { Evaluación del } \\
\text { nivel de conflicto de } \\
\text { implementación }\end{array}$ \\
\hline $\begin{array}{l}\text { Interrogante } \\
\text { fundamental (para } \\
\text { la gestión de las } \\
\text { políticas públicas) }\end{array}$ & $\begin{array}{l}\text { ¿Qué modalidades } \\
\text { (estructuras y } \\
\text { procedimientos) de } \\
\text { implementación deben } \\
\text { utilizarse a fin de } \\
\text { garantizar el mayor grado } \\
\text { de realización de los } \\
\text { objetivos oficiales? }\end{array}$ & $\begin{array}{l}\text { ¿Qué interacciones entre } \\
\text { los actores públicos y } \\
\text { privados de una red } \\
\text { de acción pública debe } \\
\text { tomarse en cuenta durante } \\
\text { la implementación para } \\
\text { que ésta sea aceptada? }\end{array}$ \\
\hline
\end{tabular}

Fuente: Tomada de SUBIRATS, Joan et al. (2008). Pp.190, a partir de Sabatier (1986).

Para Ryan (1996) también existe "una diferencia fundamental entre el modelo integrado de Winter y los marcos de análisis top-down (y algunos bottom-up) en la evaluación de los resultados de la implementación". Mientras que en "el análisis top-down se comparan los resultados de la aplicación con los objetivos del programa y metas establecidas en la política 
pública", el enfoque de Winter "se ocupa, además de los problemas abordados por el enfoque bottom-up, de los intereses de los actores en el proceso de implementación y de los resultados del cambio social". En este sentido, este enfoque evalúa "La implementación en términos de productos y resultados/ impacto".

\section{Los elementos del modelo de Winter}

El marco analítico propuesto por Winter, aplicados al análisis de la implementación de una política pública, son los siguientes: (1) el carácter del proceso de formulación de la política, antes de la ley o de la decisión de implementarla; (2) el comportamiento organizacional e interorganizacional de la implementación; (3) el comportamiento burocrático de primer nivel, y (4) la respuesta de los grupos destinatarios y otros cambios en la sociedad.

Estos elementos del modelo de Winter (1990), orientan el análisis de interpretar cómo ha sido el funcionamiento de la política pública de prevención durante los últimos diez años. Durante su estudio y análisis se pudo encontrar tanto las ventajas de un enfoque de sistema integrado en la aplicación, como las formas en que el análisis de la aplicación puede ser adaptado para comprender las influencias geográficas, institucionales, políticas y económicas del País que tienen que ver con el desempeño del sector público en materia de prevención y protección de DDHH.

\section{El carácter del proceso de formación de la política, antes de la ley o de la decisión de implementarla}

Winter destaca cuatro componentes a tener en cuenta: a) Cuanto mayor sea el conflicto, es más probable que la implementación se afectará. b) La formulación de la política es crucial en el diseño de la teoría causal, la cual permite seleccionar los instrumentos de la política que se aplicarán para lograr el cambio deseado en el comportamiento. La implementación es poco probable que tenga éxito si no hay un c) verdadero intento por resolver el problema. Por último, d) la atención prestada a la formulación de políticas es un buen indicador de la eficacia de un programa durante su ejecución. Esta atención a menudo se refleja en las relaciones directas, la coherencia política interna, los objetivos de la política, el diseño y aplicación. 


\section{El comportamiento organizacional e interorganizacional durante la implementación de la política.}

Este segundo elemento del modelo de Winter se refiere al comportamiento de las organizaciones o de las relaciones interinstitucionales con las directivas del programa. Esto incluye el grado de armonía entre los objetivos del programa y los intereses de la organización, y la cooperación entre los intereses institucionales. También es necesario considerar que el poder de organización y relaciones institucionales durante la fase de ejecución pueden haber cambiado desde la etapa de formulación, y la influencia de las coaliciones que también tenga que ser considerado. Para el caso colombiano, el comportamiento organizacional contiene la relación Nación - Territorio, es decir, las autoridades del orden nacional y las de orden departamental y municipal.

\section{El comportamiento burocrático "de primer nivel"}

En este elemento Winter centra su atención en el poder de los individuos y no tanto en la autoridad institucional y organizacional. Los burócratas "de primer nivel o de calle" tienen la capacidad de distorsionar sistemáticamente la implementación de los programas. Winter sostiene que el comportamiento "de primer nivel" para distorsionar los objetivos esperados, tiene que ver con la cultura de la organización. Por lo tanto, cambiar el comportamiento de estos burócratas requiere también un cambio en la cultura organizacional. En este contexto, incorporar la acción de los actores locales y regionales en el proceso de implementación de la política pública, determina un importante efecto de estos agentes en los resultados de la prevención.

\section{sociedad \\ La reacción de los grupos destinatarios y otros cambios en la}

Este elemento del modelo de Winter se centra en el comportamiento específico de cada grupo y las condiciones socio-económicas; en este sentido, la respuesta de los destinatarios estará influenciada por la magnitud de cambio de la conducta requerida, y la validez de la teoría causal aplicada para llevar a cabo estos cambios. Los programas deben dar cuenta de la naturaleza de los grupos objetivo. Por ejemplo, diferentes niveles educativos o socioeconómicos puede implicar diversas estrategias de implementación. 
Los grupos destinatarios son más propensos a cooperar con los programas cuando las prescripciones se ajustan a los comportamientos y normas existentes.

Nada más difícil cuando se considera la respuesta de los grupos destinatarios de un programa de prevención. Estos grupos generalmente corresponden a organizaciones sociales, sindicales y gremiales, colectivas de derechos humanos, organizaciones indígenas, de campesinos, de afrodescendientes, de líderes sociales, de defensores de derechos humanos y de organizaciones de mujeres, todas estas las principales víctimas de los actores armados. Determinar el grado de aceptación y cooperación a los mecanismos e instrumentos de prevención, constituye un indicador válido para considerar en el análisis y su efecto en la prevención de violaciones de derechos humanos.

En síntesis, el modelo de Winter integra las variables de análisis de implementación de políticas públicas que se destacan tanto en la literatura top-down como la de bottom-up. Y para efectos del análisis, los cuatro elementos de Winter se deben considerar de manera relacionada entre sí: Es así como el comportamiento de la organización y entre organizaciones se ve influenciado e influye en las acciones de los burócratas de primer nivel; estas relaciones también existen entre los grupos destinatarios y los burócratas de primer nivel. De este modo, en el examen de estos elementos hay cierta obligación de considerar el impacto de un conjunto de variables sobre las demás.

\section{Sección segunda. Configuración y evolución de la política de derechos humanos y la prevención de violaciones de DDHH/DIH en Colombia}

La exhaustiva investigación de Soto (2006), muestra que durante la administración del Presidente Andrés Pastrana se formuló una política específica en materia de derechos humanos y Derecho Internacional Humanitario, "producto de un proceso que atravesó por varias etapas y que contó, en cada una de ellas, con participantes diversos".

De acuerdo con Soto (2006):

Esteprocesoilustrauna perspectivaincrementaldelaformulación de una política, ya que constata que se nutrió, en buena medida, de la política de la administración Samper (1994-1998), cuyas 
orientaciones terminaron prevaleciendo. Sin embargo, debió enfrentar puntos de discusión críticos en su momento, que aún hoy día no se han superado por completo e inciden de manera notable en el proceso de implementación de los programas de prevención y protección de derechos humanos.

Por primera vez el tema de los derechos humanos fue encargado a un Vicepresidente de la República,La política consagró los siguientes lineamientos: a) lucha contra los grupos armados al margen de la ley; b) la seguridad de los defensores de derechos humanos y de personas amenazadas; c) atención a la población desplazada por la violencia; d) medidas particulares de impulso al Derecho Internacional Humanitario; e) impulso a la administración de justicia; y, f) un Plan Nacional de Acción de Derechos Humanos y DIH. La política finalmente expedida se estructuró alrededor de una Presentación, unos Objetivos y unas Áreas prioritarias de trabajo.

La necesidad de diseñar y poner en marcha un sistema de alerta temprana para la prevención de la ocurrencia de masacres y desplazamientos forzados, apareció registrada dentro de las "Áreas prioritarias de trabajo", y se pidió que estuviera de "conformidad con las recomendaciones de la Oficina del Alto Comisionado para las Naciones Unidas”. Pese a que se conformaron, durante toda la administración Pastrana, varios grupos de trabajo integrados por las entidades gubernamentales y estatales con responsabilidades en la protección de los derechos humanos, no se logró diseñar el Sistema.

Uno de los aspectos que destaca Soto (2006), es que desde este momento de la discusión y formulación, estuvo presente la confusión o intención de entremezclar elementos "pertenecientes a una política de seguridad o defensa" con los enfoques de políticas de derechos humanos. Al parecer, la razón que justificaba dicha apreciación respondía "a la necesidad de crear unas condiciones de seguridad propicias para el disfrute de derechos, producto de la concertación con el Ministerio de Defensa".

Así, bajo el título de "Lucha contra los grupos armados al margen de la ley", se consignaron materias tales como lucha contra la insurgencia armada, combate al secuestro, combate a los grupos de autodefensa y medidas comunes contra los grupos de autodefensa y los insurgentes" (Soto, 2006; pp. 212). Esta incorporación podría provenir del interés en poner de relieve que las acciones de los grupos armados al margen de la ley 

de derechos humanos y DIH en Colombia: un breve recuento...

eran la principal fuente de vulneración de los derechos fundamentales de los habitantes del país, situación a la cual no podía ser ajena una política de derechos humanos.

En esencia, lo que evidenció este primer conflicto de visiones (seguridad y defensa versus respeto de los derechos humanos) en esta primera etapa de la formulación de la política de derechos humanos, fue el impacto de considerar los derechos y el DIH en la cultura política y social de la acción del Estado, particularmente en los temas de seguridad, defensa y acceso a la justicia (Ministerio de la Defensa Nacional, la Cancillería, el Ministerio del Interior). En ese sentido, para enfrentar los desafíos del conflicto armado interno, los agentes estatales debían respetar los derechos humanos y el DIH y garantizar el cumplimiento en la ejecución de las medidas de prevención, control y mitigación de sus violaciones.

Desde entonces, la lucha contra la insurgencia se mantiene como el resorte de la política de seguridad y defensa adelantada por el Ejecutivo, y la política de derechos humanos se encarga de hacer seguimiento a los resultados operacionales del conflicto armado interno. La puesta en marcha del Plan Colombia fortaleció el componente de seguridad, el cual se institucionalizó con la Política de Seguridad Democrática durante los dos gobiernos del Presidente Uribe Vélez (2002-2006-2010); a partir de entonces, se impuso un cambio en la concepción de la política de derechos humanos, y muchos de los lineamientos definidos durante la administración Pastrana, quedaron relegados para darle paso a los enfoques de seguridad por encima de todo.

Durante la administración Uribe, el componente de prevención de violaciones de derechos humanos se fundamentó en la acción del sistema de alertas tempranas, del cual se esperaba que consolidara la información para la valoración oportuna del riesgo y la anticipación de eventos violentos contra la comunidad. Este Gobierno consideró al Sistema como la base de su política de prevención, y a la Seguridad Democrática como la política de derechos humanos y DIH.

Es decir, se pensó inicialmente que la puesta en práctica de la estrategia de consolidación territorial, era suficiente para lograr la prevención de violaciones de DDHH, y que bajo esa política se sometían los actores armados, logrando una reducción de las violaciones de DDHH. 
En efecto, hubo una disminución de los principales índices de violencia durante el periodo 2003 - 2009, con una notoria reducción de las masacres y de los homicidios; se logró un copamiento territorial que facilitó mayor movilidad en las carreteras, avances en la protección de la infraestructura energética, y se dio todo el proceso de desmovilización paramilitar que también condujo, en su momento, a una reducción muy fuerte de las cifras de violaciones a los DDHH.

Con base en estos supuestos, el gobierno consideró la Política de Seguridad Democrática como suficiente para prevenir, y por tanto desestimó definir una política de Derechos Humanos y de Prevención, tras considerar que la una conducía a la otra; por el contrario, la continuó a través de la fase de Consolidación (2006-2010). Tan sólo desde el pronunciamiento de la Corte Constitucional (auto 008 a la sentencia T-025) se evidenció la necesidad de estructurar una política de prevención de DDHH/DIH, y puso en discusión el sobre-reconocimiento de la Seguridad Democrática, como política de prevención.

Fue a partir de entonces que mecanismos como el SAT, comenzaron a ser considerados en la definición de los programas de prevención y atención a la población desplazada, obligando así al gobierno nacional a formalizar el CIAT de manera normativa, lo que ocurrió en el 2007.

\section{Mecanismos e instrumentos para la prevención: el Sistema General de Alertas Tempranas (SAT/CIAT)}

\section{Origen del SAT de la defensoría del pueblo y de la CIAT}

Como se mencionó anteriormente, a partir de la política de derechos humanos diseñada durante el gobierno Pastrana, se dispuso el diseño y puesta en marcha del mecanismo de alerta temprana para la prevención de desplazamientos y masacres (Soto, 2006; pp. 243). Y esto se hizo porque los hechos de violencia y la violación de derechos humanos y DIH se consideraban un grave problema público. 


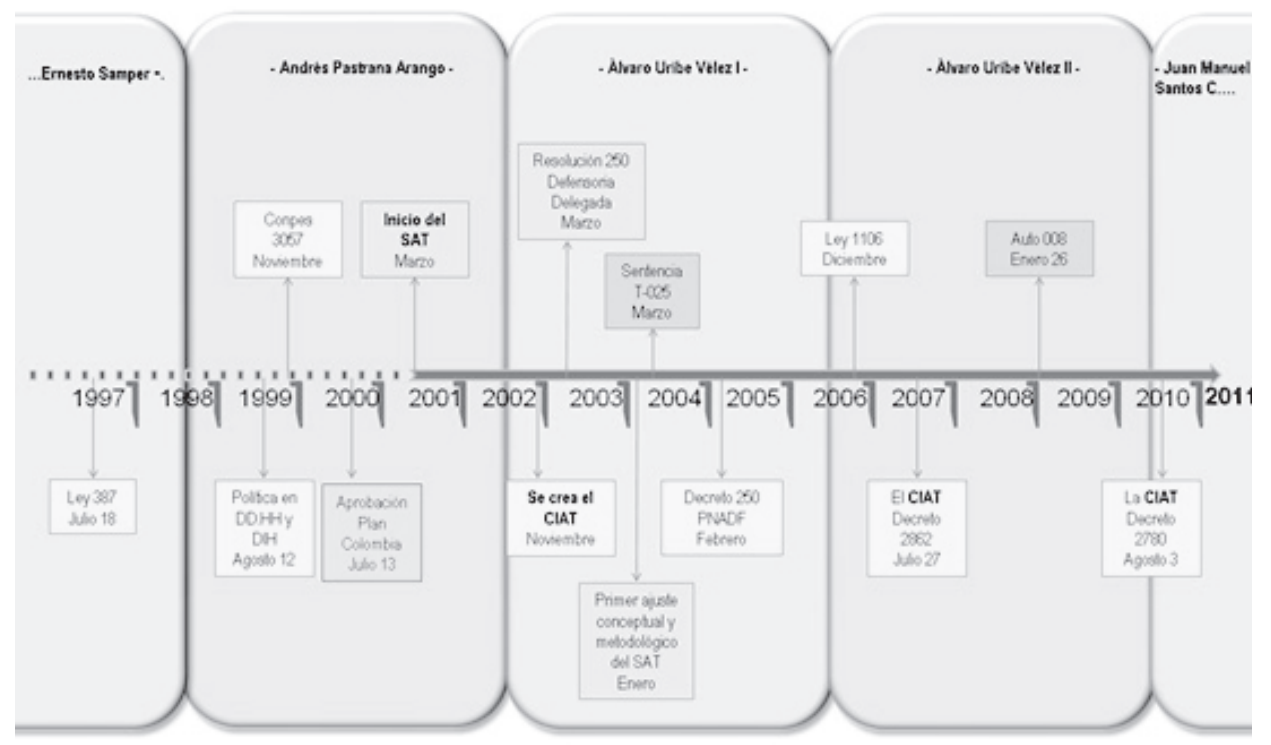

Figura 1. Proceso de institucionalización del SAT-CIAT en el contexto político nacional

Fuente: elaboración del autor con base en revisión documental y normativa.

PNADF: Plan Nacional de Atención al desplazamiento forzado

La figura 1 muestra un recuento cronológico de manera sucinta, de lo que ha sido el proceso de institucionalización e implementación del Sistema General de Alertas Tempranas (SAT- CIAT); se puede observar retrospectivamente, el resultado de un lento proceso evolutivo de lo que ha sido el interés del Estado en materia de derechos humanos (DDHH) e infracciones al Derecho Internacional Humanitario (DIH).

Fue desde el año 1995, que las autoridades colombianas con el apoyo de agencias internacionales comenzaron a discutir la necesidad de definir programas y mecanismos para la prevención de las graves violaciones masivas de derechos humanos que afectaban a la población civil (desplazamientos masivos y masacres). En 1997 se aprueba la Ley 387 que le imprime un fundamento legal a la prevención. Sin embargo, desde comienzos del año 1998 la Defensoría del Pueblo ya venía adelantando acciones hacia la identificación de los factores de riesgo derivados de conflicto armado. En 1999 con la formulación de la política de derechos humanos del Gobierno Pastrana, en noviembre se elabora el CONPES 3057 que propone la creación de un sistema de alertas tempranas en el seno de la Defensoría del Pueblo. 
Sin embargo, fue hasta marzo del año 2001 cuando se implementa el SAT cuya estrategia se basaba en la advertencia temprana y la coordinación de la respuesta en materia de protección civil y militar; a partir de este año comenzó a emitir documentos denominados Alertas Tempranas. En noviembre del año 2002, el Gobierno Nacional a través del Ministerio del Interior y de Justicia, creó el Comité Interinstitucional de Alertas Tempranas -CIAT - con la intención de concentrar y coordinar la respuesta estatal de las situaciones de riesgo advertidas por el SAT de la Defensoría del Pueblo. En conjunto, estas dos instancias configuraron lo que se podría denominar el eje de los programas de Prevención en Colombia.

El Sistema de Alertas Tempranas de la Defensoría del Pueblo fue diseñado para prevenir violaciones masivas y sistemáticas de derechos humanos y defender el derecho internacional humanitario (DIH). MANAGEMENT SYSTEMS INTERNATIONAL -MSI- (2004), afirma que "es el único sistema de este tipo que opera en el mundo" dirigido como programa público de un Estado; inició actividades como un proyecto de cooperación internacional financiado por la Agencia de los Estados Unidos para el Desarrollo Internacional -USAID-, en el seno de la Defensoría del Puebloy denominado "Sistema de Alertas Tempranas en Derechos Humanos", en el marco del Plan Colombia, para promover la implementación de un programa efectivo de derechos humanos. Su misión ha sido la de analizar el conflicto armado interno a nivel local y regional, para advertir amenazas que permitan a las autoridades concernidas implementar medidas protectoras de los derechos fundamentales de la población civil. 


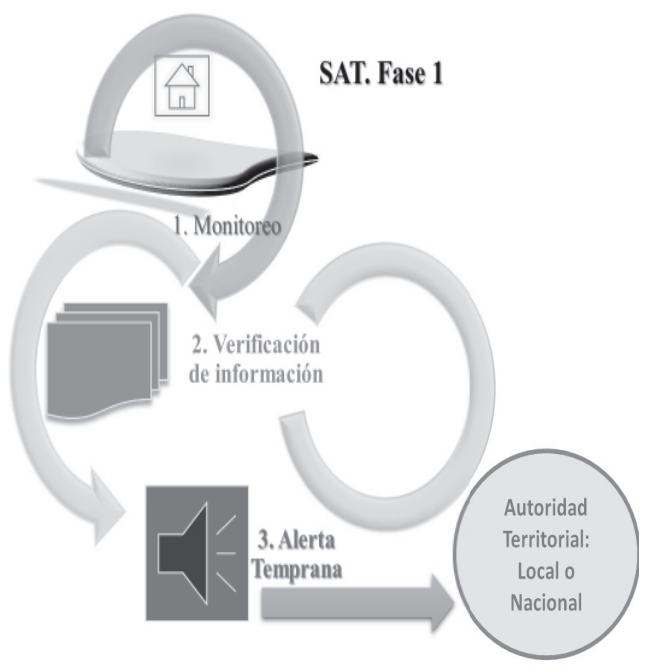

Figura 2. Sistema de Alerta Temprana Fase I (Marzo 2001- Noviembre de 2002) Fuente: SAT/USAID/MSI, (2004)

Según las evaluaciones financiadas por USAID, el SAT pasó por dos grandes momentos: una primera fase (2001- Noviembre de 2002) antes de la existencia del CIAT (Ver figura 2). Y una segunda fase a partir de diciembre del año 2002 hasta 2010 (Ver figura 3). En la primera fase, según MSI, "El sistema constituido originalmente era relativamente rápido, ágil y transparente". Las ventajas evidenciadas de este primer modelo fue que "el Delegado de la Defensoría podía verificar la información en fuentes locales y emitir alertas en un tiempo razonable. Las alertas tempranas eran públicas y concebidas en el marco nacional e internacional de los derechos humanos y el DIH".

Las evaluaciones hechas por MSI (2004) y Democracia Internacional (2009), coinciden en señalar que la desventaja de este primer modelo fue la poca incidencia que podía tener la Defensoría del Pueblo en la formulación y ejecución de políticas públicas (función del gobierno nacional), en tal sentido, la primera fase del Sistema de Alertas Tempranas fue considerada "principalmente exhortatoria". Actualmente, esta dificultad persiste.

Las contradicciones políticas sobre el surgimiento del SAT, emergieron con los hechos trágicos de Bojayá (Chocó), ya que posteriormente se supo que la Defensoría había emitido una alerta temprana anunciando a las autoridades de ataques y amenazas inminentes contra la población civil 
(MSI, 2004). Esto conllevó a un cuestionamiento desde el nivel internacional y nacional sobre el papel de las autoridades frente a las Alertas Tempranas del SAT, lo que motivó al Gobierno nacional y a las fuerzas armadas mismas, a presionar para modificar el Sistema.

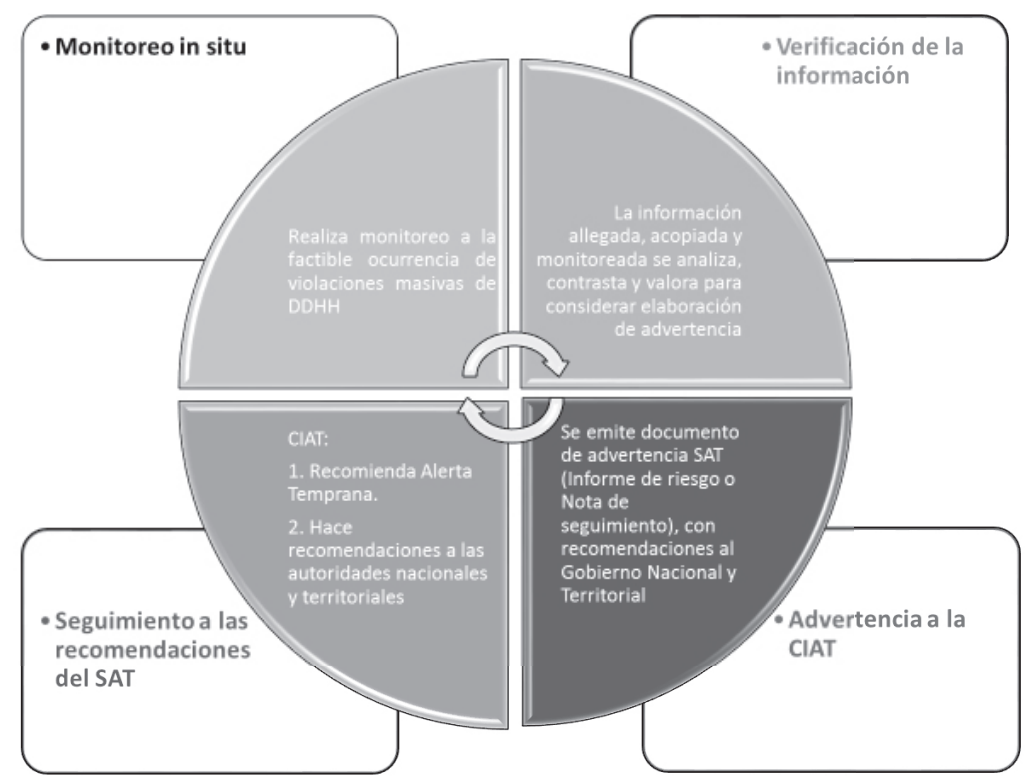

Figura 3. Sistema de Alerta Temprana Fase II (Noviembre de 2002 - 2010)

Fuente: SAT

En respuesta a las "debilidades y limitaciones del diseño" del SAT, a finales de 2002, el gobierno recién posesionado de Álvaro Uribe propuso la creación del Comité Interinstitucional de Alertas Tempranas (CIAT) para "desarrollar una respuesta estatal más coherente a los informes generados por la Defensoría del Pueblo".

En esta nueva fase del Sistema, la Defensoría no genera alertas tempranas (ver figura 3), en cambio produce Informes de riesgo. Estos Informes no son públicos ni circulan libremente, siendo enviados directamente al CIAT, cuyos miembros tienen la responsabilidad de verificar la información a través de sus representantes locales y demás contactos. Posterior a la verificación, el CIAT convoca una reunión de sus miembros y decide si es necesario emitir una alerta temprana. En los casos que la CIAT no decide emitir la alerta temprana, envía igualmente el informe de riesgo 

de derechos humanos y DIH en Colombia: un breve recuento...

a las autoridades locales competentes con una serie de recomendaciones sobre las medidas que se deben tomar.

Finalmente, MSI (2004) identificó que "el cambio de la Fase I a la Fase II, no significó una reducción apreciable en el número de violaciones sucedidas después de la emisión de una Alerta Temprana o Informe de Riesgo. Cada modelo muestra ciertas fortalezas y debilidades. Agrupadamente, los dos modelos parecen tener el mismo impacto en la prevención de violaciones masivas de derechos humanos". También recomendaba que "los Informes de Riesgo se hicieran públicos y accesibles a todos los ciudadanos y actores nacionales e internacionales que estén interesados. Una mayor apertura del sistema lo hará más efectivo y receptivo".

En lo que respecta a la consolidación de la estructura interna del SAT y del CIAT, han tenido un crecimiento exponencial durante estos 10 años; el SAT pasó de tener tres analistas, luego siete, llegando a una cobertura departamental; USAID financió progresivamente la estructura de personal toda vez que no estaba creado el Sistema dentro de la entidad. En una etapa más institucionalizada con la creación de la Delegada para la Evaluación de Riesgos de la Población Civil a consecuencia del conflicto armado, la Defensoría del Pueblo asume el financiamiento del Director, de una Secretaria y algunos costos a partir del 2004. En el 2005 se fijaron unas metas de institucionalización progresiva con el fin de que el SAT dejara de depender de la cooperación internacional, lográndose ya un 83\% de institucionalización (financiado por el presupuesto nacional); la Cooperación se ha dirigido ahora a dar un apoyo más cualificado en el tema de asistencia técnica para los ajustes metodológicos. En el caso de la CIAT, cuenta con profesionales dentro de la unidad de asuntos territoriales dedicados al análisis de los Informes de Riesgo y Notas de Seguimiento del SAT y el seguimiento de las medidas implementadas por las autoridades concernidas.

En síntesis, y desde el punto de vista de incidencia en política pública, es lo que se ha logrado construir como programa público para la prevención de violaciones de DDHH e infracciones al DIH en Colombia. Se puede afirmar, que desde el punto de vista de la cooperación, se logró la institucionalización de las dos instancias, es decir tanto del SAT como de la CIAT. El principal logro de ello es que el Gobierno nacional cuenta con una herramienta de política pública, institucionalizado y con recursos propios. 
A nivel internacional, también es considerado un modelo importante que muestra resultados y ha logrado un creciente reconocimiento, al punto que el Sistema Interamericano de Derechos Humanos, lo reconoce; la Corte Constitucional colombiana lo reconoce como el instrumento de prevención. A pesar de ello, desde 2002 y hasta el último Informe publicado a comienzos de 2011, la Oficina del Alto Comisionado para los Derechos Humanos de la ONU, insiste en pedir más apoyo para el SAT.

\section{Resultados del análisis de la implementación del programa bajo el enfoque de Winter.}

A partir del marco teórico de análisis de implementación de políticas públicas descrito y la metodología señalada, se parte de incorporar el enfoque integrado de Winter a partir de los elementos explicados en la sección anterior, para comprender las incidencias de las condiciones sociopolíticos que afectan los resultados de la implementación de la política de prevención.

\section{El proceso de formulación de la política}

La figura 4 muestra una gráfica del proceso del SGAT como mecanismo de la política pública de prevención Como se puede observar, el diseño fue pensado para que la Defensoría del Pueblo a través de su extensa red de oficinas regionales y de analistas en terreno, pudiese identificar y advertir las situaciones de riesgo a través de informes a la CIAT coordinado por el Ministerio del Interior. A partir de allí, la valoración de esta Comisión intergubernamental podría enriquecer el análisis del SAT y recomendaría al Ministerio del Interior emitir la Alerta Temprana correspondiente y ordenar la respuesta en tres tipos: Política (a cargo de las autoridades civiles como los Ministerios concernidos, Entidades Descentralizadas, Gobernaciones y Alcaldías), Policial (dirigido a la Policía Nacional) y Militar (responsables las FFMM). Se complementaba con el apoyo y seguimiento de las organizaciones internacionales de derechos humanos y organizaciones no gubernamentales nacionales, quienes contribuirían con su gestión de seguimiento, denuncia y fortalecimiento técnico a profundizar los enfoques de prevención y la implementación de políticas, programas y proyectos dirigidos a superar los factores de riesgo y las condiciones de vulnerabilidad social en tales territorios alertados. 

de derechos humanos y DIH en Colombia: un breve recuento...

Como se pudo describir, el inicio del proceso de diseño y formulación de la política se dio en medio de un contexto de gravísimas violaciones a los DDHH e infracciones al DIH, a finales de la década de los noventa. Sin embargo, el cambio en las dinámicas del conflicto genera a su vez un proceso de ampliación de los escenarios de trabajo del SAT. Es así como se empieza a ampliar la mirada a otras conductas violatorias de DDHH que era necesario advertir a través de Alertas Tempranas: Minas Antipersonal, ataques armados, homicidios selectivos dirigidos a líderes, dirigentes sociales, representantes de organizaciones de población desplazada; desapariciones forzadas, reclutamiento forzado, violencia sexual, amenazas, extorsiones, toma de rehenes, entre otras.

La transformación del proceso, con la creación del CIAT, encontró diversosobstáculosquefueron percibidos poralgunosactorescomouna forma de cooptar el trabajo que venía haciendo la Defensoría del Pueblo desde una visión de DDHH. Esta visión enfrentaba el enorme peso que tenía en ese momento la ejecución de la Política de Seguridad Democrática del Gobierno de Uribe y que incluso comprometía a muchos agentes del Estado vinculados a las FFMM, por sus acciones de connivencia con actores armados ilegales, omisión ante las amenazas y riesgos advertidos o violaciones directas de derechos humanos.

Asimismo, el grueso del poder military de gobierno, vieron amenazadas sus prioridades en materia de seguridad y estrategia militar contrainsurgente así como de la agenda política planeada, ante las contingencias de intervención que representaban las alertas tempranas provenientes de denuncias y quejas desde la sociedad civil y publicadas por la Defensoría del Pueblo (ente de control del Estado en materia de derechos humanos) sobre amenazas que acontecían en territorios de la periferia nacional (como los Montes de María), cuya intervención era consideraba de altos costos ante la baja o casi mínima importancia económica y social en el contexto nacional. 


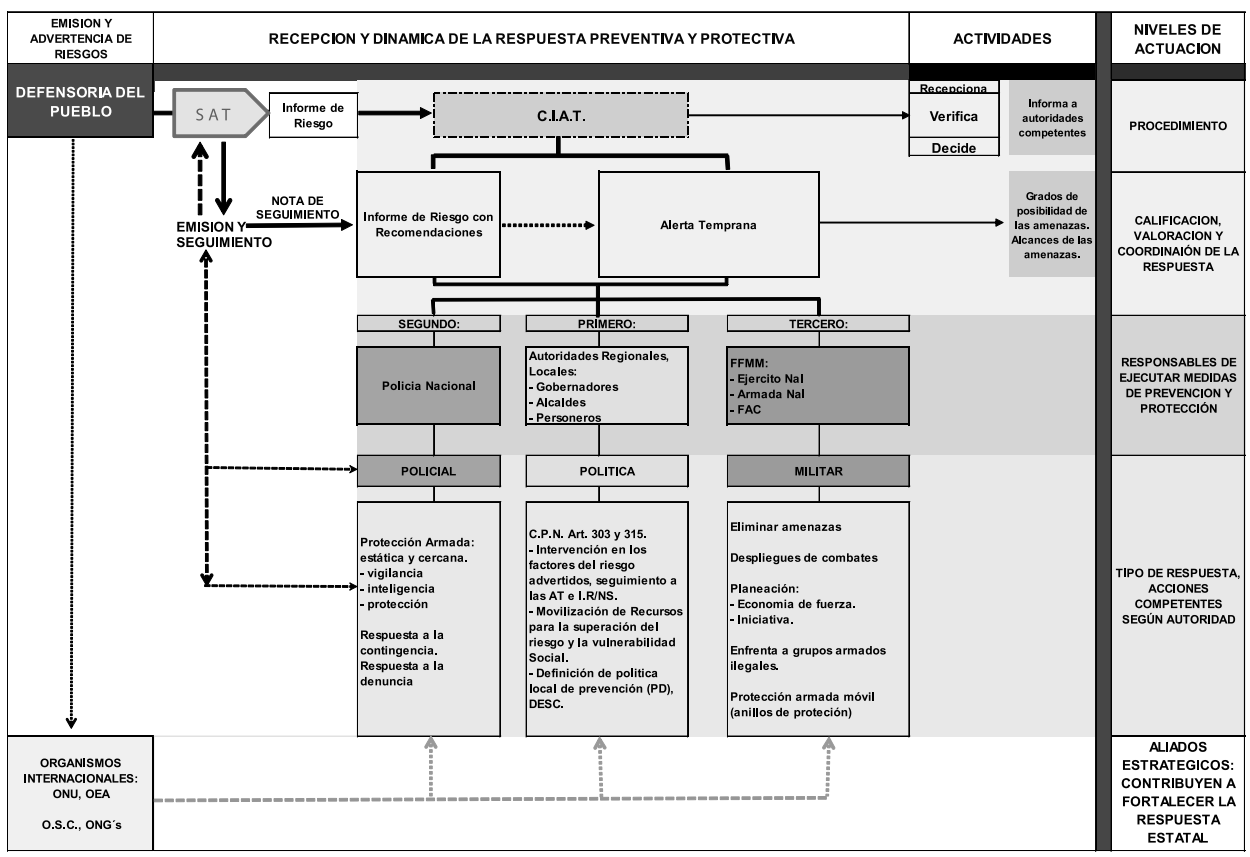

Figura 4. Esquema General del SGAT. Advertencia y Tipo de Respuesta.

Fuente: elaboración del autor, basado en documentos institucionales de DP-SAT, USAID-MSD, CIAT.

El resultado de lo anterior se expresó al interior del CIAT, dadas las dos concepciones que se encuentran frente al tema de la prevención; por un lado la DP presenta un enfoque de carácter humanitario basado en el respeto a los DDHH, en tanto que la visión gubernamental basa su mirada en un marco de seguridad y orden público orientada por la aplicación de la política de Seguridad Democrática en el territorio considerando aspectos de distancia, densidad poblacional y recursos económicos estratégicos.

Sumado a este escenario de incoherencia interna, el funcionamiento del sistema encontró grandes resistencias entre autoridades locales y fuerza militares al no realizarse un proceso previo pedagógico de información y sensibilización con los actores implicados respecto a las responsabilidades del Estado en materia de prevención y de cómo las Alertas Tempranas e Informes de Riesgo contribuían en este propósito. Este vacío generó una estigmatización de los instrumentos de prevención que desde entonces han sido vistos como denuncias, quejas o cuestionamientos a la labor de los actores locales (Autoridades regionales y locales y fuerza pública), afectando 
notoriamente la implementación del programa público de prevención, tal como se analizará más adelante.

Otrovacíoenestemomento de transformación delaimplementación del programa lo constituyó la ausencia al interior del CIAT de reglamentaciones internas y metodologías de valoración de los Informes de Riesgo que permitieran la evaluación objetiva de los mismos para determinar si se convertían o no en AT, dejando así la posibilidad de que múltiples intereses cruzaran la definición de este proceso. El peso de las orientaciones del gobierno de Uribe y su política central de Seguridad Democrática contribuyeron a generar una "Macrocefalia" de las fuerzas militares sobre el poder civil en los procesos de aplicación de la política pública de prevención en las zonas de mayor intensidad del conflicto armado, dado que el análisis de las AT que se emitieron, privilegiaron la visión de orden público (Zona de Rehabilitación y Consolidación) sobre los DDHH y las respuestas militares primaron sobre las acciones de las autoridades civiles, las cuales delegan su responsabilidad y capacidad de respuesta en el poder militar. Respuesta además que está mediada por la capacidad de los recursos con que cuentan las fuerzas militares para mantener la seguridad en las carreteras, proteger los oleoductos, las torres de energía, entre otra infraestructura.

Esta respuesta inmediata que privilegia la visión militar, en muchos territorios provocó un cambio en los efectos esperados con la emisión de las AT, puesto que se deja de lado la concepción de la prevención como una responsabilidad del Estado en su conjunto que amerita un proceso integral de respuesta, en donde además de la fuerza pública deben concurrir los esfuerzos de las instituciones civiles con el fin de disminuir los factores de riesgo de carácter estructural o vulnerabilidades que afectan a la población civil afectada por el conflicto armado. Desde esta perspectiva se privilegian entonces las respuestas de carácter reactivo frente a las acciones de prevención, dirigidas a garantizar la explotación de los recursos energéticos, en tanto que las condiciones generadoras de conflicto no se desactivan y los factores del riesgo para la población civil permanecen.

Otro de los problemas que se derivan de la etapa de formulación, es que el SAT fue diseñado principalmente para advertir sobre las violaciones cometidasporlosgruposarmadosilegalesysólopuedenseñalarindirectamente aquellas violaciones potencialmente cometidas por actores del estado. Como tal, "ni el SAT ni el CIAT fueron diseñados para abordar adecuadamente 
las violaciones por parte de actores del estado". Este aspecto fue evaluado por Democracia Internacional (2009), y consideró que "representa una falla muy seria cuando se compara con otros tipos de metodologías de alerta temprana en otras situaciones de conflicto en diferentes países, en donde todos los actores - estatales y no estatales - son monitoreados, y todas las violaciones potenciales y actuales son tomadas en cuenta".

En algunos departamentos éste aspecto representa una falla muy seria, si se consideran los efectos de las actuaciones de miembros de la Fuerza Pública en la población civil, que se ha visto reflejada en las múltiples denuncias por procesos de ejecuciones extrajudiciales (conocidos por la opinión pública como "falsos positivos") durante la aplicación de la política de Seguridad Democrática, las capturas masivas de presuntas personas vinculadas con los grupos guerrilleros y que posteriormente los fiscales y jueces deben dejar en libertad por falta de pruebas. 

de derechos humanos y DIH en Colombia: un breve recuento...

Tabla 2

Problemas frente a la Formulación de la política y niveles de afectación

\begin{tabular}{|c|c|c|c|c|}
\hline \multirow{2}{*}{$\begin{array}{l}\text { ELEMENTOS } \\
\text { DE WINTER }\end{array}$} & \multirow{2}{*}{ PROBLEMAS } & \multicolumn{3}{|c|}{ NIVELES DE AFECTACIÓN } \\
\hline & & NAL & DPTAL & LOCAL \\
\hline \multirow{3}{*}{$\begin{array}{c}\text { Formulación de } \\
\text { la política }\end{array}$} & $\begin{array}{l}\text { La tensión suscitada } \\
\text { por las deliberaciones } \\
\text { entre seguridad y } \\
\text { derechos humanos, con } \\
\text { la creación del CIAT } \\
\text { derivó en una concepción } \\
\text { sesgada de la prevención } \\
\text { que se ha traducido en } \\
\text { resistencias para actuar } \\
\text { preventivamente. }\end{array}$ & & & \\
\hline & $\begin{array}{c}\text { No hubo procesos } \\
\text { pedagógicos de } \\
\text { información y } \\
\text { sensibilización sobre } \\
\text { las responsabilidades } \\
\text { del Estado en materia } \\
\text { de prevención y como } \\
\text { las AT e Informes de } \\
\text { Riesgo contribuían a } \\
\text { este propósito, lo cual } \\
\text { se tradujo en confusión } \\
\text { e indiferencia hacia la } \\
\text { toma de decisiones para } \\
\text { prevenir. }\end{array}$ & & & \\
\hline & $\begin{array}{l}\text { Contexto mediado por } \\
\text { principios de Seguridad } \\
\text { Democrática que se } \\
\text { confunde y se interpreta } \\
\text { desde el gobierno } \\
\text { nacional con la política de } \\
\text { prevención y de DDHH, lo } \\
\text { cual le imprime un mayor } \\
\text { peso en las decisiones de } \\
\text { las Fuerzas Militares. }\end{array}$ & & & \\
\hline
\end{tabular}




\begin{tabular}{|c|c|c|c|c|}
\hline \multirow{2}{*}{$\begin{array}{l}\text { ELEMENTOS } \\
\text { DE WINTER }\end{array}$} & \multirow{2}{*}{ PROBLEMAS } & \multicolumn{3}{|c|}{ NIVELES DE AFECTACIÓN } \\
\hline & & NAL & DPTAL & LOCAL \\
\hline \multirow{3}{*}{$\begin{array}{l}\text { Formulación de } \\
\text { la política }\end{array}$} & $\begin{array}{l}\text { Ausencia de reglamentos } \\
\text { al interior del CIAT que } \\
\text { permitiesen la evaluación } \\
\text { objetiva de los Informes } \\
\text { de Riesgo para la emisión } \\
\text { o no de AT. }\end{array}$ & & & \\
\hline & $\begin{array}{c}\text { No se consideró } \\
\text { los riesgos que las } \\
\text { actuaciones de agentes del } \\
\text { Estado podían tener en la } \\
\text { población civil, generando } \\
\text { confusión y omisión frente } \\
\text { a este tipo de riesgos. }\end{array}$ & & & \\
\hline & $\begin{array}{c}\text { El SGAT, no se ha } \\
\text { articulado a una lógica } \\
\text { de sistema enmarcado en } \\
\text { una política pública de } \\
\text { prevención y de derechos } \\
\text { humanos, lo cual propicia } \\
\text { la desarticulación y } \\
\text { descoordinación en los } \\
\text { demás ámbitos del Estado. }\end{array}$ & & & \\
\hline
\end{tabular}

Fuente: Creación del autor.

Otro de los aspectos del proceso de formulación del SGAT, fue que en su diseño no se contó con un mecanismo de evaluación y seguimiento, incluso de control ciudadano. Este aspecto se consideró como dado dentro de la estructura del Estado (FGN, PGN, CGR). Quizás también porque se trataba de un programa novedoso cuyo financiamiento se correspondía a la cooperación internacional. La inclusión de la PGN fue una decisión posterior y aún presenta reticencias para participar en una instancia de gobierno, como es la CIAT. Esto refleja un vacio frente a lo idealizado (modelo de la figura 4) en el funcionamiento del SGAT. En muchos territorios la gestión institucional de la PGN, es muy distante al seguimiento y control de las medidas en el tema de la prevención, tomadas por el Estado en este contexto. 

de derechos humanos y DIH en Colombia: un breve recuento...

\section{El comportamiento organizacional e interorganizacional de la imple- mentación}

Como ya se dijo, el contexto nacional en el que se comenzó a concebirse la política pública de prevención de violaciones de DDHH estaba mediado por una fuerte discusión entre los enfoques de DDHH y Seguridad. Si bien el SAT durante el año 2001 había avanzado en la propuesta de construcción de una política pública de prevención que contemplaba la creación de un Sistema Nacional de Prevención, esta propuesta se frustró con la llegada del nuevo Gobierno en agosto de 2002, y el proyecto de decreto que buscaba reglamentar un Sistema Nacional de Prevención, no alcanzó a ser firmado por el saliente presidente Pastrana. Esta tendencia política sumada a la forma y composición del CIAT, influyó fuertemente durante los primeros años en las decisiones del CIAT al momento de emitir o no una AT dado que las decisiones tendían a tomarse con fundamento en el orden público y no en la protección humanitaria. Situación que se veía potenciada porque en el marco de una política nacional basada en el concepto de seguridad, el papel de las fuerzas armadas era central y la emisión de AT, se interpretaba como un cuestionamiento a su efectividad en el control territorial o como un insumo más para la inteligencia militar o de conocer las intenciones del enemigo. Las decisiones entonces se centraron en evaluar la veracidad de los Informes de Riesgo emitidos por el SAT, perdiendo el objetivo central para el que fue creado la CIAT el cual era impulsar la respuesta coordinada y efectiva del Estado en materia de prevención y hacer un seguimiento a los efectos o impactos de la misma.

Se reconocen sin embargo algunos avances, durante estos nueve años de estudio de la política pública de prevención, producto de las recomendaciones de organismos de cooperación internacional como OACNUDH y USAID, el Auto 008 de 2009 a la Sentencia T-025 de 2004 de la Corte Constitucional, entre otras disposiciones legales, que han contribuido a que se definan protocolos, procedimientos y metodologías para el análisis objetivo de los informes de riesgo y la activación del CIAT frente a la llegada de un Informe de Riesgo del SAT. Se efectuaron reuniones periódicas con tiempos más cortos de convocatoria en relación a la emisión del informe de riesgo, se propició la interlocución de las autoridades locales y regionales a través de video conferencias; se amplió la participación a otras dependencias del Estado que deben atender recomendaciones de tipo integral, por ejemplo: ICBF, Fiscalía 
General de la Nación, INCODER, Programa Presidencial contra MAP (PAICMA), Programa Presidencial para la Prevención del reclutamiento forzado y demás programas; y también, en ocasiones, se extendió invitación a algunas agencias internacionales. También se amplió el campo de proposición en cuanto a las recomendaciones del SAT para ser acogidas por las autoridades. Y se realizó la descentralización en el seguimiento a las recomendaciones del informe de riesgo y de la CIAT, a través de reuniones que se efectuaban en las zonas donde el SAT advirtió riesgos y donde las autoridades presentaron sus informes de las acciones, de las medidas y de los impactos de esas acciones en materia de prevención y mitigación del riesgo de manera pública.

Sin embargo, se identificó que le faltó mucha más capacidad a la Secretaría Técnica de la CIAT para asumir el liderazgo en la coordinación de respuestas integrales frente a las situaciones de riesgo, lo cual aunado a la ausencia de un marco normativo en materia de política pública de prevención hace que aún hoy la respuesta del Estado sea tardía, reactiva, desarticulada e insuficiente. Tardía, porque el proceso de análisis del informe de riesgo no tiene una respuesta inmediata, tampoco se conocen los tiempos que se toman las autoridades para emprender acciones frente a los mismos y no se hizo seguimiento a esas actuaciones. Es reactiva, porque aún se impone la respuesta militar y de policía para enfrentar la situación de riesgo, pero no hubo una respuesta integral y sostenida del Estado para atender aspectos estructurales de las condiciones de vulnerabilidad de las poblaciones afectadas, por lo tanto la respuesta se quedó en el nivel asistencial siendo por tanto insuficiente. Y finalmente fue desarticulada, porque al no existir una política de derechos humanos, la prevención adolecía de una estructura de sistema nacional que integrara todos los esfuerzos y programas de prevención y protección del Estado que en ese momento se llevaban de manera independiente. Tampoco se realizó un seguimiento efectivo a las acciones propuestas por el nivel nacional y subnacional.

Se debe destacar que a nivel intra organizacional, Acción Social emprendió transformaciones en sus diferentes niveles para asumir el tema de prevención; a la luz del Decreto 250 del 2005 crea el Grupo de Prevención y Emergencias en respuesta a la necesidad de integrar el Consejo Nacional de Atención a la Población Desplazada. Este grupo desarrolló internamente una labor de difusión de la importancia de la prevención al interior de la entidad, 

de derechos humanos y DIH en Colombia: un breve recuento...

diseñó protocolos de actuación frente a los informes de riesgo, creó una mesa interna de prevención, tenía en cuenta los Informes de riesgo y las Alertas Tempranas en el proceso de focalización de sus programas y creó indicadores para la evaluación de sus actuaciones. Este grupo se ubicaba a nivel nacional y en algunas oficinas territoriales de Acción Social. Sin embargo, fue una iniciativa interna de Acción Social, que no estaba integrada a una acción articulada y coordinada con el resto de instancias de prevención del Estado.

Si bien, en el nivel nacional el tema de la prevención comenzó a posicionarse un poco más, con la ayuda del nivel técnico que conmina al nivel político para que le de la importancia y la preponderancia que tiene, a nivel local aún faltaba bastante para que esto se realizara, tal como el análisis del comportamiento de los "burócratas de primer nivel", demostrará. Sin embargo, este aspecto evidencia el bajo o nulo rol de la sociedad civil en la corresponsabilidad de la prevención; no se ha logrado construir o formalizar un marco de participación local que tenga injerencia en los análisis y decisiones en la gestión de riesgos y amenazas. La polarización ha hecho casi que imposible la participación en estos escenarios ante la desconfianza, temor y miedo de lo que se diga frente a los hechos reales.

Uno de los mayores vacios que presenta en el relacionamiento interorganizacional es la débil y casi ausente presencia de la Procuraduría General de la Nación -PGN-; ésta institución de control disciplinario del Estado no está bien integrada a las acciones del SAT/CIAT y no participa en las deliberaciones de la CIAT, a pesar que en el Decreto 2780 de 2010 aparece como invitado permanente.

La mayor incidencia de la PGN está relacionado con los casos que involucran a agentes del Estado; el curso de acción correcto es informar directamente a la Procuraduría General de la Nación, quien debe investigar y tomar las acciones disciplinarias necesarias; este conducto, sin embargo, no se encuentra vinculado al sistema SAT/CIAT, ya que las infracciones y violaciones por parte de actores del estado no son consideradas asuntos a nivel de la CIAT, y en caso de serlo, se convertiría en un escenario muy problemático ya que sus miembros vienen del gobierno y de la fuerza pública del estado. El Tabla 3 sintetiza los problemas planteados. 
Tabla 3

Problemas frente al Comportamiento organizacional e interorganizacional

\begin{tabular}{|c|c|c|c|c|}
\hline \multirow{2}{*}{$\begin{array}{c}\text { ELEMENTOS DE } \\
\text { WINTER }\end{array}$} & \multirow{2}{*}{ PROBLEMAS } & \multicolumn{3}{|c|}{ NIVELES DE AFECTACIÓN } \\
\hline & & NAL & DPTAL & LOCAL \\
\hline \multirow[t]{2}{*}{$\begin{array}{c}\text { Comportamiento } \\
\text { organizacional }\end{array}$} & $\begin{array}{c}\text { La falta de voluntad } \\
\text { política evidencia que } \\
\text { organizacionalmente } \\
\text { la Dirección Nacional } \\
\text { (MIJ-CIAT), } \\
\text { Departamental y } \\
\text { Municipal, tienen } \\
\text { capacidad discrecional } \\
\text { para ejecutar o no las } \\
\text { medidas de prevención } \\
\text { y/o mitigación del } \\
\text { riesgo. Esto no permite } \\
\text { una actuación objetiva } \\
\text { para prevenir. }\end{array}$ & & & \\
\hline & $\begin{array}{c}\text { No se ha } \\
\text { institucionalizado } \\
\text { un mecanismo } \\
\text { de respuesta, } \\
\text { independiente de } \\
\text { la decisión política } \\
\text { de alguna de las } \\
\text { autoridades, lo que } \\
\text { lleva a la prevención a } \\
\text { depender de decisiones } \\
\text { y criterios políticos y } \\
\text { personales. }\end{array}$ & & & \\
\hline
\end{tabular}



de derechos humanos y DIH en Colombia: un breve recuento...

\begin{tabular}{|c|c|c|c|c|}
\hline \multirow{2}{*}{$\begin{array}{c}\text { ELEMENTOS DE } \\
\text { WINTER }\end{array}$} & \multirow{2}{*}{ PROBLEMAS } & \multicolumn{3}{|c|}{ NIVELES DE AFECTACIÓN } \\
\hline & & NAL & DPTAL & LOCAL \\
\hline & $\begin{array}{l}\text { Confusión en el } \\
\text { objetivo último del } \\
\text { CIAT (impulso de la } \\
\text { respuesta coordinada } \\
\text { y efectiva en materia } \\
\text { de prevención y } \\
\text { seguimiento) que hace } \\
\text { que su actuación se } \\
\text { quede en la evaluación } \\
\text { de los Informes de } \\
\text { Riesgo y no en la } \\
\text { trascendencia de las } \\
\text { accionesde prevención. }\end{array}$ & & & \\
\hline $\begin{array}{c}\text { Comportamiento } \\
\text { organizacional }\end{array}$ & $\begin{array}{l}\text { La "Macrocefalia" de } \\
\text { las fuerzas militares } \\
\text { en la aplicación de } \\
\text { medidas de prevención } \\
\text { derivadas del enfoque } \\
\text { de seguridad y orden } \\
\text { público, que lleva a } \\
\text { que las Autoridades } \\
\text { civiles deleguen su } \\
\text { responsabilidad de } \\
\text { actuación a favor de } \\
\text { la fuerza pública, } \\
\text { limitando el tipo } \\
\text { de intervención y } \\
\text { prevención. }\end{array}$ & & & \\
\hline & $\begin{array}{c}\text { La respuesta no es } \\
\text { integral, mediada por } \\
\text { enfoque de seguridad, } \\
\text { no se atacan las } \\
\text { vulnerabilidades, y } \\
\text { por tanto persisten los } \\
\text { factores de riesgo. }\end{array}$ & & & \\
\hline
\end{tabular}




\begin{tabular}{|c|c|c|c|c|}
\hline \multirow{2}{*}{$\begin{array}{c}\text { ELEMENTOS DE } \\
\text { WINTER }\end{array}$} & \multirow{2}{*}{ PROBLEMAS } & \multicolumn{3}{|c|}{ NIVELES DE AFECTACIÓN } \\
\hline & & NAL & DPTAL & LOCAL \\
\hline \multirow{3}{*}{$\begin{array}{c}\text { Comportamiento } \\
\text { organizacional }\end{array}$} & $\begin{array}{c}\text { Fuerzas Militares y } \\
\text { Civiles perciben las } \\
\text { AT y los Informes } \\
\text { de Riesgo como } \\
\text { cuestionamiento } \\
\text { a su labor, lo que } \\
\text { conlleva a actuaciones } \\
\text { desinteresadas } \\
\text { y parcializadas, } \\
\text { según conveniencia } \\
\text { institucional. }\end{array}$ & & & \\
\hline & $\begin{array}{c}\text { Existe una debilidad } \\
\text { en la Secretaria } \\
\text { Técnica para asumir } \\
\text { el liderazgo en la } \\
\text { coordinación de la } \\
\text { respuesta, la cual } \\
\text { ante el marco de una } \\
\text { política pública de } \\
\text { prevención, genera } \\
\text { una respuesta de } \\
\text { Estado tardía, reactiva, } \\
\text { desarticulada e } \\
\text { insuficiente. }\end{array}$ & & & \\
\hline & $\begin{array}{c}\text { Problemas de } \\
\text { interacción entre } \\
\text { Acción Social y las } \\
\text { demás entidades del } \\
\text { Gobierno nacional } \\
\text { y territorial, lo cual } \\
\text { no garantiza el } \\
\text { cumplimiento de la } \\
\text { prevención in situ. } \\
\text { Limitaciones de su } \\
\text { actuación en el ámbito } \\
\text { territorial. }\end{array}$ & & & \\
\hline
\end{tabular}



de derechos humanos y DIH en Colombia: un breve recuento...

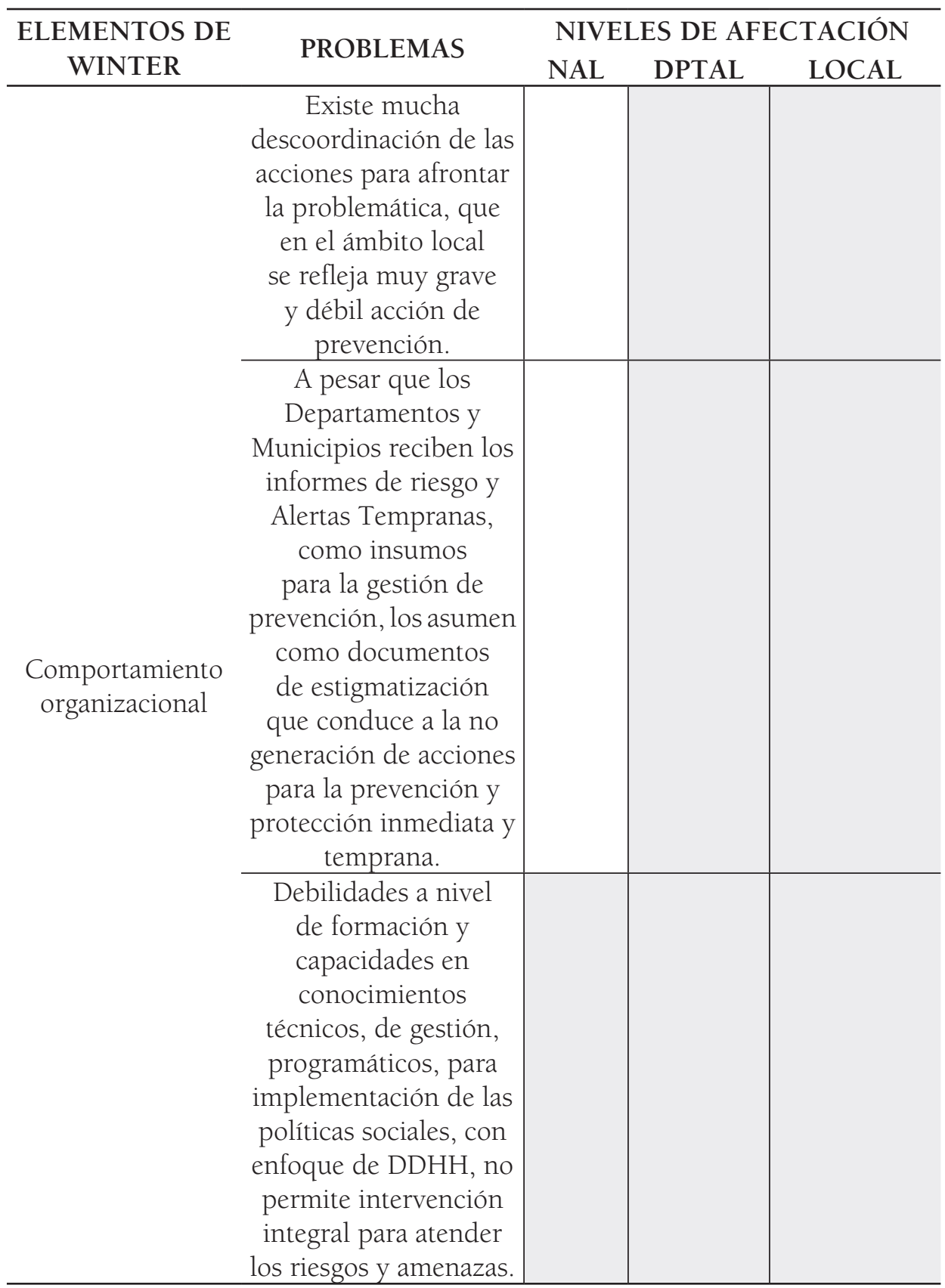

Fuente: Creación del autor. 


\section{Los burócratas "de primer nivel" o de primera línea de atención}

Siguiendo a Winter (1990), es necesario centrar la atención en el poder de los individuos (poder saber, analizar, decidir, hacer) para llevar a cabo la implementación de las acciones requeridas, en este caso, prevención de violaciones de DDHH/DIH. Ahora bien, al analizar a los funcionarios como actores de primera línea, hay que considerar que en departamentos y municipios existen muchas carencias a nivel de la formación y capacidades en conocimientos técnicos, de gestión, programáticos; para la generación e implementación de políticas que, adecuadas al contexto, incorporen enfoques de derechos humanos, diferenciales (ciclo de vida, género, diversidad cultural, entre otros), y que consideren seriamente la responsabilidad del Estado en materia de prevención y protección. Asimismo, que se promueva y construya un desarrollo con perspectiva local $\mathrm{y}$ de frente a los retos del proceso globalizador, en especial en territorios marginales (¿Qué tipo de desarrollo? ¿Social, económico?, ¿Para quién?). Sumado a esto se encuentran las restricciones en términos de acceso a recursos, conocimientos, conectividad vial, peso político de la región a nivel nacional y las profundas repercusiones en materia política, social, cultural y económica que el conflicto armado en su larga duración ha dejado entre la población y en el territorio.

Con estos condicionantes y en este contexto, no es de extrañar que si bien la autoridad local (alcalde o gobernador) se constituye en el máximo responsable del orden público a nivel territorial, la realidad demuestra que no han asumido esta responsabilidad, dado que en este campo el liderazgo es del Ejército y la Policía Nacional. De esta manera, los Informes de Riesgo o las AT son discutidos en los Consejos de Seguridad que, derivados de la política de Seguridad Democrática, generalmente se realizaban a nivel territorial cada semana; es decir, los instrumentos de prevención no movilizan la respuesta coordinada de las autoridades frente a los riesgos sobre la población civil, sino que la conveniencia o no de dichos documentos, se constituyen en uno de los puntos a tratar en dichos escenarios.

Ahora bien, la implementación de la respuesta en el nivel regional y municipal, estaba mediada tal como se ha reseñado, por el énfasis que se hace en la concepción de seguridad y orden público frente al enfoque de DDHH; en este sentido el papel de las fuerzas militares es determinante en la actitud que se asume frente a los Informes de riesgo y las Alertas Tempranas, 

de derechos humanos y DIH en Colombia: un breve recuento...

así como en las acciones que de ellas se derivaban dado que se interpretaba erróneamente que estos informes "evalúan" la gestión de la fuerza pública o las autoridades locales generando frente a los mismos conductas de negación o resistencia frente a lo que allí se plantea o recomienda. En este aspecto, el modelo ideal que propone el SGAT (figura 4), de responder en tres frentes: primero, Político; segundo, Policial; y tercero, Militar; aquí no encuentra expresión, sino que por el contrario se sobredimensionan el segundo y tercer tipo de respuesta (policial y militar) sobre la dimensión de política pública en áreas relacionadas con los DESC, que realmente contribuyen a superar los factores de riesgo y las condiciones de vulnerabilidad.

Las perspectivas de estos actores han contribuido a hacer que las AT o Informes de Riesgo sean vistos como instrumentos de "estigmatización del territorio", dado que los intereses de las autoridades locales se centran en promover una imagen de tranquilidad y paz como condición del desarrollo territorial. Si bien las condiciones de seguridad mejoraron respecto a los años anteriores, la situación distaba mucho de ser "el paraíso" que muchos promulgaban. Ahora bien, la negación de las autoridades del orden local y departamental frente a las situaciones de riesgo detectadas, la actitud de displicencia, de decir "aquí no pasa nada", de tranquilidad frente a las situaciones que se presentan, hace que las recomendaciones promovidas desde la CIAT no sean asumidas, como se había dicho desde una manera integral, coordinada y sostenida en el tiempo.

Esinteresante observar cómo desde la percepción de los actores del nivel nacional, departamental y local, uno de los principales factores que interfiere en la respuesta frente a las situaciones de riesgo detectadas lo constituye la "falta de voluntad política", concepto que sin embargo, es interpretado de manera diferente por cada uno de ellos. Desde el nivel nacional se percibe que a nivel territorial, las autoridades del orden municipal y departamental no tienen la "voluntad política" para reconocer las situaciones de riesgo y actuar en consecuencia frente a las mismas.

Y desde el nivel local, se percibe que los niveles departamental y nacional no tienen la "voluntad política" para acompañar, prestar asesoría técnica, apoyar con recursos, al municipio a enfrentar las situaciones de riesgo que al fin y al cabo ocurren es en su jurisdicción, por lo tanto "se reacciona con lo que se tiene". De esta manera, las acciones se han venido 
realizando de manera descoordinada entre los diferentes niveles, afectando con ello la eficacia de las medidas tomadas a nivel territorial.

La negación de la realidad, la falta de voluntad política, la descoordinación entre las acciones institucionales emprendidas por los diferentes niveles sumado a la ausencia de una política nacional en materia de prevención han contribuido a que el tema no haga parte de la Agenda local, la prevención fue considerada "el patito feo de la casa", por tanto no es considerada ni asumida como un componente de la política pública local y se encuentra ausente en los programas de gobierno y los planes de desarrollo de los mandatarios locales, siendo de esta manera su efectividad casi nula tal como lo demuestran, tanto la continuidad de las situaciones de violación de derechos para los habitantes del departamento, así como las evaluaciones que de este proceso hacen las OSC.

\section{Valoración de las acciones de las autoridades territoriales}

La caracterización de las respuestas y acciones de las autoridades del orden nacional, departamental y municipal a los Informes de Riesgos y Alertas Tempranas emitidas, se analizan a partir de los registros de la CIAT en una Matriz de seguimiento. Se puede decir que en la mayoría de las Recomendaciones emitidas desde la CIAT, hicieron énfasis en la Coordinación, tanto entre Autoridades Civiles y Militares, como entre Entidades Territoriales (Alcaldías y Gobernaciones) con instituciones descentralizadas y programas del MIJ, Vicepresidencia, etc.

Un aspecto que se puede deducir de estos reportes es que existe una multiplicidad de escenarios de reunión o convocatoria: comités, mesas, programas, etc., que no cuentan de una instancia de análisis y coordinación que permita la articulación como sistema. En la realidad local de estos escenarios, la mayoría de veces van las mismas personas que repiten los mismos procesos y percepciones del problema.

Los informes dan cuenta de datos cuantitativos en términos de inversión de número de talleres o beneficiarios, pero no de procesos sociales o de continuidades en las acciones. Primero, la información se limita a las acciones, no a los impactos de las acciones; y segundo, no hay retroalimentación de arriba abajo. 

de derechos humanos y DIH en Colombia: un breve recuento...

\section{Tabla 4}

Problemas sobre el comportamiento de burócratas de primer nivel

\begin{tabular}{|c|c|c|c|c|}
\hline \multirow{2}{*}{$\begin{array}{l}\text { ELEMENTOS } \\
\text { DE WINTER }\end{array}$} & \multirow[t]{2}{*}{ PROBLEMAS } & \multicolumn{3}{|c|}{$\begin{array}{c}\text { NIVELES DE } \\
\text { AFECTACIÓN }\end{array}$} \\
\hline & & NAL & DPTAL & LOCAL \\
\hline \multirow{4}{*}{$\begin{array}{c}\text { Burócratas a nivel } \\
\text { de calle }\end{array}$} & $\begin{array}{c}\text { Existen graves problemas en la } \\
\text { cadena de mando para responder } \\
\text { a las Alertas Tempranas; una } \\
\text { cosa es el comportamiento } \\
\text { de los funcionarios del orden } \\
\text { Departamental y otra en el } \\
\text { municipal, mediado por intereses } \\
\text { políticos. }\end{array}$ & & & \\
\hline & $\begin{array}{c}\text { Los burócratas a nivel de calle } \\
\text { no tienen una buena formación } \\
\text { en derechos humanos y DIH } \\
\text { que les permita distinguir de } \\
\text { mejor manera las violaciones } \\
\text { e infracciones respectivas y a } \\
\text { sí mismo la importancia de su } \\
\text { actuación frente a la prevención. }\end{array}$ & & & \\
\hline & $\begin{array}{l}\text { Dualidad en funcionarios de } \\
\text { atención de la prevención: } \\
\text { orden público y prevención } \\
\text { humanitaria, lo cual conlleva } \\
\text { a acciones sin mucha } \\
\text { responsabilidad frente al reto de } \\
\text { la prevención y protección. }\end{array}$ & & & \\
\hline & $\begin{array}{l}\text { Los burócratas de calle tienen } \\
\text { una margen de actuación muy } \\
\text { limitado frente a los riesgos } \\
\text { que encarna su labor en medio } \\
\text { de actores armados y conflicto: } \\
\text { misión médica, educación, } \\
\text { atención niñez, étnicos, } \\
\text { saneamiento básico, entre otras. } \\
\text { Bajo y limitado impacto de la } \\
\text { prevención por temor y miedo de } \\
\text { los funcionarios. }\end{array}$ & & & \\
\hline
\end{tabular}


Los burócratas de calle también tienen desconfianza y temor en gobernantes y fuerza pública, lo cual conlleva a una discrecionalidad con bajo impacto preventivo.

Existe desobediencia por no considerar importante cumplir las órdenes y medidas diseñadas que afecta el cumplimiento de la prevención y atención.

Están sujetos a limitantes logísticas respecto al suministro de bienes y servicios, dotación, vehículos, pagos salarios y honorarios, entre otros, que no permiten llevar a cabo las acciones para prevenir y proteger.

Muchos burócratas de calle ignoran la existencia del SAT/ CIAT y por tanto desconocen la importancia de los IR y AT. No las consideran una herramienta importante para prevenir. Consideran que los temas de DDHH/DIH son asuntos ajenos a sus funciones, propias tan sólo de la DP, PGN, Personerías, más no del ámbito de la alcaldía o la gobernación, incidiendo de manera importante en la descoordinación, desarticulación y falta de integralidad en la intervención del problema. "No es conmigo".

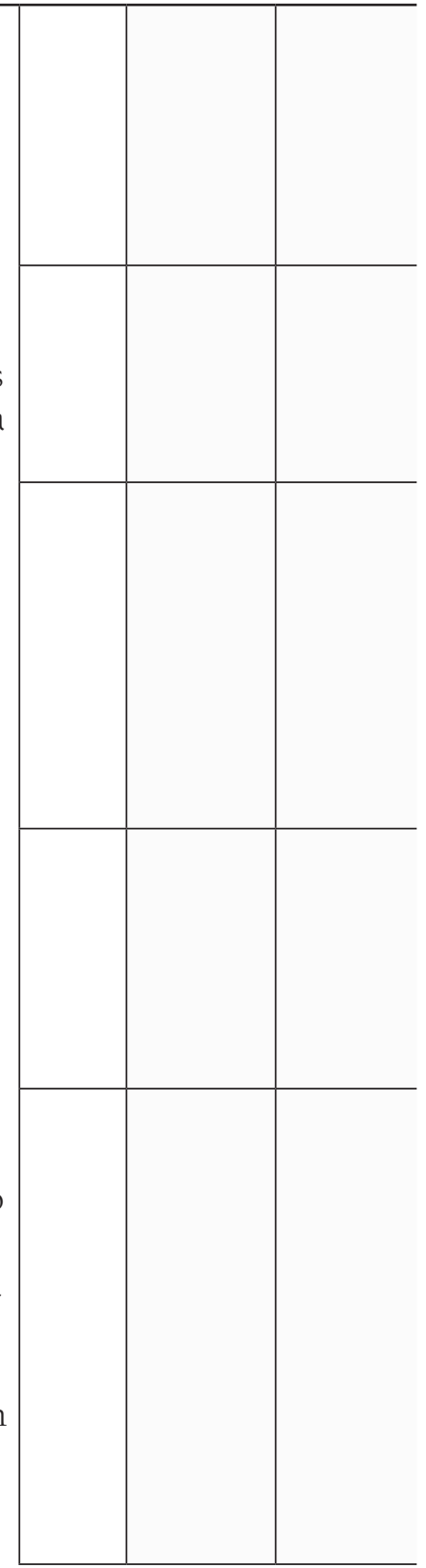




\begin{tabular}{|c|c|c|c|c|}
\hline \multirow{2}{*}{$\begin{array}{c}\text { ELEMENTOS } \\
\text { DE WINTER }\end{array}$} & \multirow[t]{2}{*}{ PROBLEMAS } & \multicolumn{3}{|c|}{$\begin{array}{c}\text { NIVELES DE } \\
\text { AFECTACIÓN }\end{array}$} \\
\hline & & NAL & DPTAL & LOCAL \\
\hline & $\begin{array}{l}\text { Negación frente a la realidad } \\
\text { expuesta en Informes de riesgo y } \\
\text { Alertas Tempranas, temor a la } \\
\text { estigmatización del territorio y } \\
\text { abandono de la inversión como } \\
\text { condición para el desarrollo. }\end{array}$ & & & \\
\hline & $\begin{array}{c}\text { El tema de la prevención no hace } \\
\text { parte de la agenda pública local } \\
\text { y por tanto los funcionarios no } \\
\text { lo consideran en la discusión } \\
\text { y formulación de los planes de } \\
\text { desarrollo. }\end{array}$ & & & \\
\hline
\end{tabular}

Fuente: Creación del Autor.

Como aspecto notorio y grave para el Programa, es que no se reportan acciones de seguimiento por parte del Ministerio Público (PGN, Personerías municipales) a pesar que se le adjudican acciones de control de las acciones según organismos competentes en prevención y vigilancia en el cumplimiento de las recomendaciones dadas por la CIAT.

El Tabla 4 sintetiza los hallazgos para este elemento de Winter aplicado.

\section{La reacción de los grupos destinatarios y otros cambios en la sociedad}

A nivel normativo es claro que no se puede hablar de Seguridad sin Derechos Humanos, pero en el caso colombiano, durante los dos períodos del gobierno de Uribe se asumió que la política de Seguridad Democrática era una política preventiva y de Derechos Humanos. Y la confusión de esta perspectiva, que privilegió las razones de seguridad nacional sobre otras consideraciones, propició de parte del gobierno nacional la negación de la existencia de un conflicto armado interno en el país para desarrollar la teoría de la "amenaza terrorista" desdibujando totalmente la grave crisis social y humanitaria que en diversos momentos fue advertida por la Corte Constitucional y otras instancias de control como la PGN. Esta perspectiva contribuyó a la estigmatización de los defensores de derechos humanos a quienes en varias ocasiones y de manera pública se les tildó de "terroristas" 
o auxiliadores de los grupos armados, generando enormes riesgos en sus derechos a la vida, la libertad e integridad personal y al libre ejercicio de su profesión, en especial en departamentos donde son señalados de ser colaboradores de los grupos armados, tanto por las organizaciones ilegales como por las mismas autoridades civiles y militares.

La polarización social derivada de estas posturas afectó considerablemente la generación de políticas en materia de prevención y DDHH tanto a nivel nacional y territorial ya que era imposible reconciliar visiones frente al conflicto armado radicalmente opuestas. Es así como frente a los efectos del conflicto armado, las organizaciones sociales, demandan respuestas que trasciendan el carácter policivo-militar que se aplica en el territorio, para generar propuestas de carácter integral que se orienten a desactivar las causas estructurales del conflicto armado.

Desdeestaperspectiva, laimplementación deunapolíticade prevención deberíaincorporarotros componentesque, además desuperarlascondiciones de vulnerabilidad de la población, permitiesen hacer efectivos los derechos a la verdad, justica y garantías de no repetición dados los altos niveles de impunidad que se viven en el territorio. Sin embargo estas reflexiones no son planteadas por las autoridades del orden departamental y local, dada su negativa a reconocer las situaciones de riesgo para la población.

Es así como se configuró una relación mediada por la desconfianza entre las autoridades y las organizaciones sociales y los bajos impactos que las medidas implementadas frente al conflicto armado durante estos diez años. Esta fue una barrera muy grande para que se pueda implementar adecuadamente un programa de prevención que convoque y vincule a todos los actores responsables del departamento, particularmente las OSC como sindicatos, ONG's, Organizaciones comunales, gremios de la producción regional y local, para que conjuntamente con autoridades se promoviera la participación y corresponsabilidad en la prevención y protección, como por ejemplo a través de Comités Locales de Prevención. 

de derechos humanos y DIH en Colombia: un breve recuento...

Tabla 5

Problemas en la Reacción de grupos destinatarios a nivel territorial

\begin{tabular}{|c|c|c|c|c|}
\hline \multirow{2}{*}{$\begin{array}{l}\text { ELEMENTOS } \\
\text { DE WINTER }\end{array}$} & \multirow{2}{*}{ PROBLEMAS } & \multicolumn{3}{|c|}{ NIVELES DE AFECTACIÓN } \\
\hline & & NAL & DPTAL & LOCAL \\
\hline \multirow{5}{*}{$\begin{array}{l}\text { Reacción grupos } \\
\text { Destinatarios }\end{array}$} & $\begin{array}{l}\begin{array}{l}\text { Estigmatización de los grupos Defensores de } \\
\text { organizaciones sociales y ONG's }\end{array} \\
\end{array}$ & & & \\
\hline & $\begin{array}{l}\text { Polarización social interfiere en la generación de políticas en } \\
\text { materia de prevención y DDHH }\end{array}$ & & & \\
\hline & $\begin{array}{l}\text { Demanda respuestas integrales para desactivar las causas } \\
\text { estructurales del conflicto armado, las cuales relacionan con la } \\
\text { riqueza del territorio Araucano. }\end{array}$ & & & \\
\hline & $\begin{array}{l}\text { Aportes de insumos a la política de prevención para superar no } \\
\text { sólo las condiciones de vulnerabilidad sino para hacer realidad los } \\
\text { derechos ala verdad, justicia y garantías de no repetición. }\end{array}$ & & & \\
\hline & Desconfianza entre autoridades y organizaciones sociales. & & & \\
\hline
\end{tabular}

Fuente: Creación Propia del autor.

En el Tabla 5, se puede sintetizar los hallazgos descritos anteriormente.

\section{Conclusiones y recomendaciones}

\section{Dentro del ámbito de la formulación de la política:}

- Los problemas que se presentaron en la etapa de formulación no permiten generar una serie de programas articulados, coordinados y en corresponsabilidad con las diferentes autoridades civiles, militares y OSC.

- Comoseobservóen los resultados, la preponderanciadel paradigma de la seguridad, en desmedro de la prevención humanitaria, está sujeta e incide en que no haya un verdadero intento de resolver el problema de violaciones de derechos e infracciones al DIH para el departamento.

- El comportamiento interorganizacional:

- La macrocefalia militar, la falta de acciones integrales, la falta de coordinación, la falta de apropiación de las distintas autoridades en los diferentes niveles y la falta de voluntad política, hace que desde que se emite una alerta temprana o informe de riesgo, el impacto de las acciones se diluya a medida que la implementación se aproxima al ámbito local, que es donde existe el riesgo y la 
amenaza de violaciones de DDHH-DIH, y por tanto es muy débil lo que en materia de prevención se implementa.

\section{Burócratas de primer nivel de atención:}

- En los burócratas de primer nivel se observa falta de perfiles adecuados en los funcionarios públicos que hace que no se identifique correctamente cuándo ocurre una violación e infracción al DIH.

- Pero además, los funcionarios también tienen miedo a actuar ante las probables represalias que los actores armados puedan dirigir hacia ellos.

- La mayoría no están de acuerdo con los diagnósticos de los IR/ AT y sienten que los problemas son mucho menores que los que advierten dichos documentos.

- Todos estos factores hacen que los burócratas de primer nivel tengan mayor discrecionalidad sobre la acción que ejecutan (u omitir su acción) y la utilicen para reducir el efecto de la prevención en el ámbito de su competencia y actividad.

\section{Reacción de los Grupos destinatarios:}

- La relación de los grupos sociales, las comunidades y personas en el contexto territorial, con el Estado y el gobierno (nacional, departamental y municipal), está mediada por el miedo, la desconfianza y por la precariedad de su condición (vulnerabilidad). Todo esto hace que sientan que todavía no hay una acción de gobierno eficaz y real que tenga como centro la protección de las personas como sujetos de derechos frente al riesgo derivado del conflicto armado.

- Consideran que todas las acciones de seguridad están dirigidas a garantizar la explotación de las riquezas del territorio. 
Reflexiones sobre la implementación de la política pública de prevención de violaciones de derechos humanos y DIH en Colombia: un breve recuento...

\section{Referencias}

ABRAMOVICH, Víctor. Una aproximación al enfoque de derechos humanos en las estrategias y políticas de desarrollo. En, Revista de la CEPAL No. 88, abril de 2006.

AGUILERA PEÑA, Mario. ELN: entre las armas y la política. En, Universidad Nacional - IEPRI. Nuestra guerra sin nombre. Transformaciones del conflicto en Colombia. Bogotá: Grupo Editorial Norma, 2005. Pp. 209-266.

AUSTIN Alexander. Early Warning and the field: A cargo cult science? En: http://www.berghof-handbook.net.

BARRERA, Cristina. Flujos decisionales en escenarios de tensión especial. En, Cristina Barrera (Comp.). Crisis y Fronteras. Relaciones fronterizas binacionales de Colombia con Venezuela y Ecuador. CEREC, CIDER, Ediciones Uniandes. Bogotá, 1989.

BARRS, Caey. Conflict Early Warning: Warning who? En: http://www.jha. ac/articles/al84.pdf

Consultado por última vez el 21 de Mayo de 2009.

BRECKE Peter. Risk Assessment Models and Early Warning Systems. En: http://bibliothek.wzb.eu/pdf/2000/p00-302.pdf

DENSCOMBE, Martyn. Good Research Guide. Buckingham, GBR: Open University Press, 2007. p127. Consultado en: http://site.ebrary. com/lib/bibliotecauniandes/Doc?id=10197064\&ppg=136

EISENHARDT, K.M., 1989. "Building theory from case study research" in Academy of Management Reviw, Volume 14, Number 4.

GAITAN GARCIA, Olga Lucía y Luis Eduardo Pérez Murcia. Prevención de violaciones de los derechos de las personas en situación de riesgo extraordinario o extremo. Obligaciones y retos del Estado colombiano. Programa de Derechos Humanos de USAID. Bogotá, 2008 .

GALVIS ORTIZ, Ligia. Comprensión de los Derechos Humanos. Una visión para el siglo XXI. Ediciones Aurora, Cuarta Edición, Bogotá, Abril de 2008.

GARFIELD, Elsie, Jairo Arboleda y Alberto Chueca. Violencia, paz sostenible y desarrollo. En, GIUGALE, Marcelo M., Olivier Lafourcade y 
Connie Luff (Editores). Colombia. Fundamentos Económicos de la Paz. Banco Mundial - Alfaomega. Bogotá, 2003. pp. 3-24.

GIRALDO CASTAÑO, Germán G. La Colonización en la Orinoquia Colombiana, Arauca 1900 - 1980. Ediciones Antropos Ltda. Bogotá D.C., Primera edición, Abril de 2006.

GUTIERREZ LEMUS, Omar Jaime. Arauca: un territorio de contrastes. En, CONTROVERSIA No. 192. Centro de Investigación y Educación Popular -CINEP-. Tercera Etapa. Junio de 2009. Pp. 41-79.

MARTINEZ CARAZO, Piedad Cristina. El Método de Estudio de Caso. Estrategia metodológica de la investigación científica. En, PENSAMIENTO Y GESTIÓN, No. 20. Universidad del Norte, pp. 165-193. 2006.

MENY, Ives y Jean Claude Thoenig. Las políticas públicas. Barcelona, Editorial Ariel S.A., 1992.

PEÑATE, Andrés. El sendero estratégico del ELN: del idealismo guevarista al clientelismo armado. En, DEAS, Malcom y Llorente, María Victoria. Reconocer la guerra para construir la paz. Bogotá, Uniandes - CEREC - Norma. Pp. 53-98.

PETERS, B. Guy \& Jon Pierre. Handbook of Public Administration. SAGE Publications. Reprinted 2005.

PROGRAMA DE LAS NACIONES UNIDAS PARA EL DESARROLLO. El conflicto, callejón con salida. Informe Nacional de Desarrollo Humano para Colombia - 2003. Bogotá, Colombia, septiembre de 2003. Páginas 79 a 95.

ROTH D., André-Noël. Políticas públicas. Formulación, implementación y evaluación. Ediciones Aurora, Bogotá, Sexta edición, 2007.

ROTH D., André-Noël. Discurso sin compromiso. La política pública de los derechos humanos en Colombia. Ediciones Aurora, Bogotá, marzo de 2006.

RYAN, Neal. Some advantages of an integrated approach to implementation analysis: a study of australian industry policy. En, Public Administration Vol. 74 Winter 1996 (737-753). Blackwell Publishers Ltd. 1996, Cambridge, USA.

SCOTT, W. Richard. Institutions and organizations. Foundations for organizational science. SAGE Publications, California, 1995 

de derechos humanos y DIH en Colombia: un breve recuento...

SISTEMA DE ALERTAS TEMPRANAS. Luz para la Vida. Masacres ocurridas en Colombia 1999. Defensoría del Pueblo, ACNUR. Bogotá.

SOTO RESTREPO, Carmen Elisa. Generación de políticas gubernamentales de derechos humanos a partir de la Constitución de 1991. Bogotá: Ediciones Uniandes, 2006.

WEIMER, David L. y Aidan R. Vining. Policy analisys. Concepts and practice. New Jersey, Pearson - Prentice Hall. Fourth edition, 2005.

WIESNER DURAN, Eduardo. La efectividad de las políticas públicas en Colombia. Un análisis neo institucional. DNP - Tercer Mundo Editores. Bogotá, septiembre de 1998.

WINTER, Soren C. Implementation. Introducción. En, PETERS, B. Guy \& Jon Pierre. Handbook of Public Administration. SAGE Publications. 2005. pp. 205-211.

WINTER, Soren C. Implementation Perspectives: Status and Reconsideration. En, PETERS, B. Guy \& Jon Pierre. Handbook of Public Administration. SAGE Publications. 2005. pp. 212-222.

YIN, Robert. K. Case Study Research: Design and Methods. Third Edition. Applied Social Research Methods Series. Volume 5. Sage Publications. 2003.

\section{DOCUMENTOS NORMATIVOS Y DE POLÍTICA PÚBLICA}

Documento CONPES 2804 de 1995

Documento CONPES No 3057 de 1999.

CONSEJO DE ESTADO. Sala de Consulta y Servicio Civil. Radicación No. 1.969 de 2009. Referencia: "Rama Ejecutiva del Poder Público. Nivel Nacional, Sector Central. Consejos superiores, Organismos consultivos o coordinadores, Comisiones Intersectoriales. La Defensoría del Pueblo y el Sistema Nacional de Alertas Tempranas. El comité Interinstitucional de Alertas Tempranas, CIAT".

Resolución 250 de 2003 emitida por la Defensoría del Pueblo establece como función específica de la Defensoría Delegada para la Evaluación de Riesgos de la Población Civil como consecuencia del Conflicto Armado,

Decreto 250 de 2005, por el cual se expide el Plan Nacional para la Atención Integral a la Población Desplazada por la Violencia y se dictan otras disposiciones. 
Decreto 2111 de 1987

Decreto 1680 de 1991

Ley 387 de 1997

Ley 1106 de diciembre 22 de 2006 por medio de la cual se prorroga la vigencia de la Ley 418 de 1997 prorrogada y modificada por las Leyes 548 de 1999 y 782 de 2002 y se modifican algunas de sus disposiciones.

Sentencia T-025 de 2004

Auto 08 de 2009, de seguimiento a la sentencia T-025 de 2004 de la Honorable Corte Constitucional.

Decreto 2780 de octubre de 2010, el cual también modificó la denominación, composición y estructura de la CIAT.

REPUBLICA DE COLOMBIA. DEPARTAMENTO NACIONAL DE PLA-

NEACION. Plan Nacional de Desarrollo 1998 - 2002, Cambio para construir la paz. Tomos I y II.

REPUBLICA DE COLOMBIA. DEPARTAMENTO NACIONAL DE PLA-

NEACION. Plan Nacional de Desarrollo 2002 - 2006, Hacia un

Estado comunitario.

REPUBLICA DE COLOMBIA. DEPARTAMENTO NACIONAL DE PLA-

NEACION. Plan Nacional de Desarrollo 2006 - 2010, Estado Comunitario: Desarrollo para Todos.

REPUBLICA DE COLOMBIA. VICEPRESIDENCIA DE LA REPUBLICA.

PROGRAMA PRESIDENCIAL DE DERECHOS HUMANOS Y DE-

RECHO INTERNACIONAL HUMANITARIO. Política de derechos humanos y Derecho Internacional Humanitario.

REPUBLICA DE COLOMBIA. PRESIDENCIA DE LA REPUBLICA. Informe del Presidente Uribe al Congreso de la República 2010.

VICEPRESIDENCIA DE LA REPUBLICA. Política de Derechos Humanos y Derecho Internacional Humanitario, 2006.

Documentos institucionales internos de trabajo

\section{usaid/msd}

ESTRATEGIA DE PREVENCION DEL PROGRAMA DE DERECHOS HUMANOS DE USAID, Operado por MSD. 2009. Documento interno de discusión. 

de derechos humanos y DIH en Colombia: un breve recuento...

OFICINA DEL PROCURADOR GENERAL -RIG-. Auditoría del Programa de Derechos Humanos de USAID/Colombia. Informe de Auditoría No. 1-514-09-007-P. Marzo 06 de 2009. San Salvador, El Salvador. Documento Interno de USAID.

MANAGEMENTSYSTEMSINTERNATIONAL-MSI-.EvaluacióndelSistema de Alertas Tempranas en Colombia. Washington, Noviembre 3 de 2004.

DEMOCRACY INTERNATIONAL INC. Evaluación del programa de derechos humanos de USAID en Colombia. Informe final, enero 2009.

\section{DEFENSORÍA DEL PUEBLO}

DEFENSORÍA DEL PUEBLO. Información General Sistema de Alertas Tempranas. Delegada para la valoración de riesgos de la población civil como consecuencia del conflicto armado. Bogotá, 2004

DEFENSORÍA DEL PUEBLO. Delegada para la Prevención de Riesgos de la Población Civil como consecuencia del Conflicto Armado, SAT. Lineamientos de política pública para la prevención de violaciones masivas de derechos humanos en el conflicto armado. Bogotá D.C., Noviembre de 2003.

DEFENSORÍA DEL PUEBLO. Información General Sistema de Alertas Tempranas. Delegada para la Evaluación de riesgos de la población civil como consecuencia del conflicto armado. Bogotá, 2004.

DEFENSORÍA DEL PUEBLO. Entronque del Sistema de Alertas Tempranas en la Defensoría del Pueblo. Bogotá D.C., Febrero de 2002. Documento de trabajo interno del SAT.

\section{SITIOS WEB CONSULTADOS}

PROGRAMA DE DERECHOS HUMANOS DE USAID. http://www.programaddhhcolombia.org/

http://www.disaster-info.net/desplazados/legislacion/06-02-04SENTENCIAT-025-041.pdf

http://www.pnud.org.co/img_upload/9056f18133669868elcc381983d50faa/capitulo_3.pdf

http://www.derechoshumanos.gov.co/Observatorio/EstadisticasxDepto/2009-2010/01-09/arauca.pdf 